\title{
Phonon-assisted relaxation kinetics of statistically-degenerate excitons in high-quality quantum wells
}

\author{
A.V. Sorokof and A.L. Ivanov \\ Department of Physics and Astronomy, Cardiff University, Cardiff CF24 3YB, Wales, United Kingdom
}

\begin{abstract}
Acoustic-phonon-assisted thermalization kinetics of excitons in quantum wells (QWs) is developed for small concentrations of particles, $\rho_{2 \mathrm{D}} \lesssim 10^{9} \mathrm{~cm}^{-1}$, when particle-particle interaction can be neglected while Bose-Einstein statistics already strongly influences the relaxation processes at low temperatures. In this case thermalization of QW excitons occurs through nonequilibrium states and is given by the following scenario. During the first transient stage, which lasts a few characteristic scattering times, the correlations with an initial distribution of QW excitons disappear. The next, adiabatic stage of thermalization usually takes many characteristic scattering times, depends only upon two control parameters, the lattice temperature $T_{b}$ and the degeneracy temperature $T_{0} \propto \rho_{2 \mathrm{D}}$, and is characterized by a quasi-equilibrated distribution of high-energy QW excitons with effective temperature $T(t)$. We show that the thermalization law of high-energy particles is given by $\delta T(t)=$ $T(t)-T_{b} \propto e^{-\lambda_{0} t} / t$, where $\lambda_{0}$ is a marginal value of the continuous eigenvalue spectrum of the linearized kinetics. By analyzing the linearized phonon-assisted kinetics of statistically-degenerate QW excitons, we study the dependence $\lambda_{0}=\lambda_{0}\left(T_{b}, T_{0}\right)$. Our numerical estimates refer to high quality GaAs and ZnSe QWs. Finally, we propose a special design of GaAs-based microcavities, which considerably weakens the bottleneck effect in relaxation of excitons (polaritons) and allows us to optimize the acoustic-phonon-assisted thermalization processes.
\end{abstract}

PACS numbers: 78.66-w, 72.10.Di, 63.20.Kr

\section{INTRODUCTION}

The formation, resonant or phonon-assisted, of QW excitons and their following relaxation towards a final (quasi-) equilibrium thermodynamic state at low lattice temperature $T_{b}$ are the subject of numerous

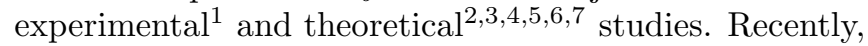
relaxation thermodynamics has been formulated and developed in order to analyze how Bose-Einstein statistics of high-density QW excitons influences the phononassisted thermalization processes 1 The above thermodynamics assumes a strong, dominant exciton-exciton scattering and, therefore, relaxation through quasiequilibrium thermodynamic states. The relaxation thermodynamics has successfully been applied to model the $\rho_{2 \mathrm{D}}$-dependent thermalization and photoluminescence kinetics observed in early experiments 10 with highdensity excitops $\left(5 \times 10^{9} \mathrm{~cm}^{-2} \leq \rho_{2 \mathrm{D}} \leq 10^{11} \mathrm{~cm}^{-2}\right)$ in GaAs QWs.

The long-lived indirect excitons in high-quality GaAs/AlGaAs coupled QWs provide a unique opportunity for studying quantum degeneracy in a system of two-dimensional bosons. In this case, the long radiative lifetimes of indirect excitons allows the system to cool down to temperatures where the dilute exciton gas be-

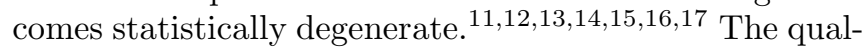
ity of present day GaAs/AlGaAs coupled QWs has been considerably improved in comparisgn with those used

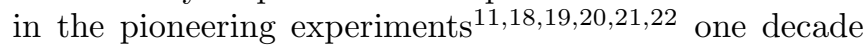
ago. Furthermore, the very recent magneto-optical experiment 23 clearly indicate that the in-plane momentum $\hbar \mathbf{k}_{\|}$of indirect excitons is a well-defined quantum number in high-quality GaAs/AlGaAs coupled QWs.

Thermalization of hot photoexcited excitons down to the temperature of the cold lattice occurs mainly via scattering by thermal bulk longitudinal acoustic (LA) phonons and is much more efficient for quasi-twodimensional (quasi-2D) systems as compared to bulk semiconductors. This follows from the relaxation of momentum conservation in the $z$-direction (the QW growth direction) for quasi-2D systems: the ground-state mode $\mathbf{k}_{\|}=\mathbf{0}$, i.e., the energy state $E=0$, couples to the continuum energy states $E \geq E_{0}$, rather than to the single energy state $E=E_{0}=2 M_{x} v_{s}^{2}\left(v_{s}\right.$ is the longitudinal sound velocity and $M_{x}$ is the in-plane translational mass of excitons) as occurs in bulk materials. As a result, the LA-phonon assisted kinetics of QW excitons becomes dominant at $\rho_{2 \mathrm{D}} \lesssim 1-3 \times 10^{9} \mathrm{~cm}^{-2}$ : in this case exciton-exciton scattering can be neglected while Bose-Einstein (BE) statistics already strongly influences the relaxation process at low temperatures Crossover from classical to quantum statistics occurs near the degeneracy temperature $k_{B} T_{0}=2 \pi \hbar^{2} \rho_{2 \mathrm{D}} /\left(g M_{x}\right)$, where $g$ is the spin degeneracy factor. For $\rho_{2 \mathrm{D}}=3 \times 10^{9}$ $\mathrm{cm}^{-2}$ the degeneracy temperature of indirect excitons in GaAs/AlGaAs coupled QWs is $T_{0}=0.79 \mathrm{~K}$. This estimate refers to $g=1$, which can be achieved in the $b$-type GaAs/AlGaAs coupled QWs by applying a static magnetic field $\mathbf{H} \| \mathbf{z} .13$ Note that the very recent experiments 15 deal with GaAs/AlGaAs coupled QWs at extremely low cryostat temperature $T_{b}=0.05 \mathrm{~K}$.

In this paper we study analytically and model numerically the acoustic-phonon-assisted relaxation kinetics of statistically-degenerate QW-excitons at low densities. The recent experiment,24,25 allow us to visualize, by means of LO-phonon-assisted emission, the LAphonon-assisted kinetics of quasi-2D excitons in $\mathrm{ZnSe}$ QWs and, in particular, to prove that for $\rho_{2 \mathrm{D}} \lesssim 10^{9} \mathrm{~cm}^{-2}$ 
the above kinetics indeed occurs through non-equilibrinm distributions of QW excitons. In experiments $12.13,14,25$ the concentration of BE-degenerate indirect excitons in GaAs/AlGaAs coupled QWs usually varies from $\rho_{2 \mathrm{D}} \gtrsim$ $10^{10} \mathrm{~cm}^{-2}$ at the very end of an optical excitation pulse to $\rho_{2 \mathrm{D}} \lesssim 10^{8} \mathrm{~cm}^{-2}$ at large delay times $t \gtrsim 50 \mathrm{~ns}$ after the optical excitation. Thus, thermalization of the indirect excitons at large delay times cannot be described within the relaxation thermodynamics 8 and does need a separate theoretical analysis.

The classical Boltzmann kinetic equation has been generalized in order to include quantum statistics by Uehling and Uhlenbeck.26 The relevant quantum kinetic equation for spatially-homogeneous dilute system of statistically-degenerate quasi-2D excitons coupled to bulk LA-phonons is

$$
\begin{aligned}
\frac{\partial}{\partial t} N_{\mathbf{k}_{\|}}= & -\frac{2 \pi}{\hbar} \sum_{\mathbf{q}}\left|M\left(q, q_{z}\right)\right|^{2}\left\{\left[N_{\mathbf{k}_{\|}}\left(1+n_{\mathbf{q}}^{\mathrm{ph}}\right)\left(1+N_{\mathbf{k}_{\|}-\mathbf{q}_{\|}}\right)-\left(1+N_{\mathbf{k}_{\|}}\right) n_{\mathbf{q}^{\mathrm{ph}}}^{\mathrm{ph}} N_{\mathbf{k}_{\|}-\mathbf{q}_{\|}}\right] \delta\left(E_{\mathbf{k}_{\|}}-E_{\mathbf{k}_{\|}-\mathbf{q}_{\|}}-\hbar q v_{s}\right)\right. \\
& \left.+\left[N_{\mathbf{k}_{\|}} n_{\mathbf{q}}^{\mathrm{ph}}\left(1+N_{\mathbf{k}_{\|}+\mathbf{q}_{\|}}\right)-\left(1+N_{\mathbf{k}_{\|}}\right)\left(1+n_{\mathbf{q}^{\mathrm{ph}}}^{\mathrm{ph}}\right) N_{\mathbf{k}_{\|}+\mathbf{q}_{\|}}\right] \delta\left(E_{\mathbf{k}_{\|}}-E_{\mathbf{k}_{\|}+\mathbf{q}_{\|}}+\hbar q v_{s}\right)\right\}
\end{aligned}
$$

where $N_{\mathbf{k}_{\|}}$and $n_{\mathbf{q}}^{\mathrm{ph}}$ are the occupation numbers of exciton in-plane mode $\mathbf{k}_{\|}$and phonon bulk mode $\mathbf{q}=$ $\left\{\mathbf{q}_{\|}, q_{z}\right\}$, respectively, and $\mathbf{q}_{\|}$is the in-plane projection of q. The terms in the first and second square brackets on the right-hand-side (r.h.s.) of Eq. (1.1) describe the Stokes and anti-Stokes LA-phonon-assisted scattering processes, respectively. The acoustical phonons are assumed to be in a thermal equilibrium at the bath temperature $T_{b}$ 目 The matrix element is given by $M\left(q, q_{z}\right)=$ $\left[\left(D_{x}^{2} \hbar q\right) /\left(2 \rho v_{s} V\right)\right]^{1 / 2} F_{z}\left(q_{z} L_{z} / 2\right)$, where $\rho$ is the crystal mass density, $D_{x}$ is the deformation potential of exciton - LA-phonon interaction, $L_{z}$ is the thickness of a QW, and $V$ is the volume. The form-factor $F_{z}(\chi)=$ $[\sin (\chi) / \chi]\left[e^{i \chi} /\left(1-\chi^{2} / \pi^{2}\right)\right]$ refers to an infinite rectangular confinement potential.27 The latter function describes the relaxation of the momentum conservation law in the $z$-direction and characterizes a spectral band of bulk LAphonons, which effectively interact with QW excitons. Note that Eq. (1.1) is valid only for the kinetic stage of thermalization, in pefore a low-temperature collective state of exciton 28 22 30 . 31 builds up.

The main aim of our work is to study the fundamental features of the acoustic phonon-assisted thermalization kinetics of QW excitons from initial strongly nonequilibrium $N_{\mathbf{k}_{\|}}(t=0)$ towards the final equilibrium distribution with well-developed Bose-Einstein statistics, when $N_{\mathbf{k}_{\|}=\mathbf{0}}^{0} \gtrsim 1$. Our numerical simulations of the LAphonon-assisted kinetics clearly demonstrate that after the first transient, which lasts a few characteristic scattering times, a slow adiabatic stage of thermalization builds up (see Fig. 1). This stage is characterized by a quasi-equilibrium distribution of high-energy QW excitons with effective temperature $T(t)=T_{b}+\delta T(t)$ and is independent of the initial distribution at $t=0$. The adiabatic stage lasts many characteristic scattering times and arises due to the need to populate the low-energy in-plane modes with $N_{\mathbf{k}_{\|} \simeq 0} \gtrsim 1$ in the presence of effective suppression of the stimulated scattering processes (e.g., an intense incoming, Stokes flux of excitons into the ground-state mode $\mathbf{k}_{\|}=0$ is nearly compensated by the outgoing, anti-Stokes scattering out of the state $\mathbf{k}_{\|}=0$ ). In order to describe the adiabatic stage of relaxation, we find a generic solution of the quantum kinetic Eq. (1.1). While the generic solution we calculate is different from that derived in Ref. 40 for the phonon-assisted kinetics of bulk excitons at $T_{b} \leq T_{c}$ ( $T_{c}$ is the critical temperature for Bose-Einstein condensation of excitons in bulk semiconductors), similar to this case it depends only on two control parameters of the system, $T_{0}$ and $T_{b}$. Furthermore, the only gross information from a particular shape of the initial distribution at $t=0$ is absorbed by the start time $t_{c}$ of the adiabatic stage of thermalization. This is shown in the Fig. 11(a), where we vary the parameters of the initial Gaussian distribution. In turn, the first transient depends upon initial distribution and lasts only a few scattering times $\tau_{\mathrm{sc}}$, as illustrated in Fig. 1(b).

Because at any nonzero bath temperature $T_{b}>0$ the occupation number of the ground-state mode $N_{\mathbf{k}_{\|}=\mathbf{0}}^{0}=$ $\exp \left(T_{0} / T_{b}\right)-1$ is finite, the adiabatic stage ends up with linearized kinetics. The latter kinetics refers to the last, exponential stage of thermalization at $t \rightarrow \infty$ and is characterized by $N_{\mathbf{k}_{\|}=\mathbf{0}}(t)-N_{\mathbf{k}_{\|=\mathbf{0}}^{0}}^{0} \propto T(t)-T_{b} \propto e^{-\lambda_{0} t}$. For statistically-degenerate $\mathrm{QW}$ excitons the thermalization time $\tau_{\text {th }}=1 / \lambda_{0}\left(T_{b}, T_{0}\right)$ reaches its smallest values at bath temperatures $E_{0} / k_{B} \ll T_{b} \lesssim T_{0}$. While the above inequality does not usually hold in the experiments with statistically-degenerate indinect excitons in GaAs/AlGaAs coupled QWs, 12,13.44.15.16.17 we describe a special design of GaAs-based microcavities (MCs) for optimization of the LA-phonon-assisted thermalization kinetics of low-density MC polaritons. In the proposed microcavities with a large positive detuning between the cavity and QW exciton modes, the MC polaritons have radiative lifetimes on a $100 \mathrm{ps}-1 \mathrm{~ns}$ time scale, so that the phonon-assisted relaxation towards well-developed BoseEinstein statistics with large occupation numbers can optically be visualized. Thus, the MC design we discuss is an interesting alternative to the semiconductor micro- 

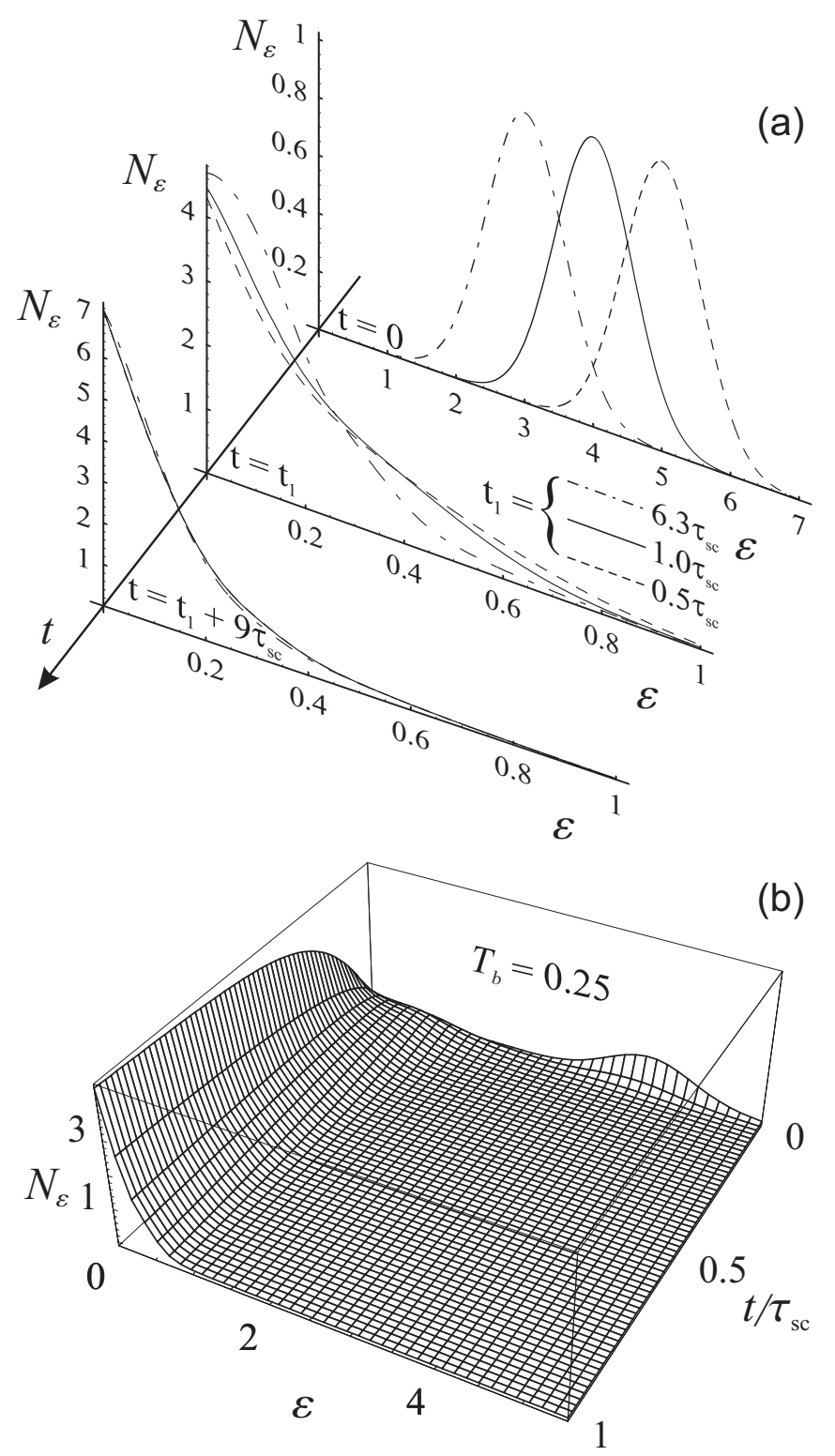

FIG. 1: (a) Transient relaxation towards the adiabatic stage of evolution calculated for various initial Gaussian distributions $N_{\varepsilon}(t=0) \propto e^{-15.625(\varepsilon-\bar{\varepsilon})}$, where $\varepsilon=\hbar^{2} \mathbf{k}_{\|}^{2} /\left(2 M_{x} E_{0}\right)$, and parameter $\bar{\varepsilon}=3$ (dash-dotted lines), 4 (solid lines), and 5 (dashed lines). The gross dependence of evolution upon initial conditions is absorbed by time $t_{1} \simeq t_{c}$. (b) The first stage of LA-phonon-assisted relaxation $\left(t \lesssim \tau_{\text {sc }}\right)$ for a particular distribution of indirect excitons with $\bar{\varepsilon}=4$. In both plots $T_{b}=0.25$ and $T_{0}=1.14$. The dimensionless values, time in $\tau_{\mathrm{sc}}$ and energy/temperature in $E_{0}$, can easily be rescaled to the dimensional units by using $E_{0}=33 \mu \mathrm{eV}$ and $\tau_{\mathrm{sc}}=41$ ns for GaAs/AlGaAs coupled QWs, and $E_{0}=162 \mu \mathrm{eV}$ and $\tau_{\mathrm{sc}}=2.7 \mathrm{~ns}$ relevant to $\mathrm{ZnSe} \mathrm{QWs}$. cavities with zero detuning between the cavity mode and QW excitons, where huge nonclassical occupation numbers of the low-energy been observed $32,33,34,35,36,37$

In numerical evaluations we use $M_{x}=0.21 m_{0}$, $v_{s}=3.7 \times 10^{5} \mathrm{~cm} / \mathrm{s}$, and $D_{x}=15.5 \mathrm{eV}$, relevant to GaAs/AlGaAs coupled QWs, and $M_{x}=0.86 m_{0}$, $v_{s}=4.1 \times 10^{5} \mathrm{~cm} / \mathrm{s}$, and $D_{x}=6.9 \mathrm{eV}$, relevant to single ZnSe QWs, respectively $\left(m_{0}\right.$ is the free electron mass). Note that the disorder-induced scattering and localization processes, not included in our model, are relatively strong in up-to-date ZnSe-based QWs and require a separate analysis. 238 However, in the very recent work 39 the first fabrication of high-quality $\mathrm{MgS} / \mathrm{ZnSe} / \mathrm{MgS} \mathrm{QWs}$ with less than $1 \mathrm{ML}$ fluctuations of the well width and, therefore, with extremely low inhomogeneous broadening has been reported.

In Sec. II, the Boltzmann equation is adapted in order to formulate the acoustic-phonon-assisted kinetics of statistically-degenerate QW excitons. We also discuss some approximations for the form-factor $F_{z}(\chi)$, which describes the relaxation of momentum conservation in scattering of QW excitons by bulk LA-phonons.

In Sec. III, we find the generic solution of the acousticphonon-assisted kinetics from a strongly nonequilibrium initial distribution of QW excitons $N_{\varepsilon}(t=0)$ towards a final Bose-Einstein distribution $N_{\varepsilon}^{0}$ with large occupation numbers of the low-energy states, $N_{\varepsilon \simeq 0}^{0} \gtrsim 1$, where $\varepsilon$ is the dimensionless energy defined by $\varepsilon=\hbar^{2} \mathbf{k}_{\|}^{2} /\left(2 M_{x} E_{0}\right)$. The generic solution is independent of a particular shape of the initial distribution and describes the adiabatic stage of thermalization, which starts at $t=t_{c}$ in a few characteristic scattering times after $t=0$. We show that at $t \gg t_{c}$ thermalization of high-energy QW excitons is given by $\delta T(t) \propto e^{-\lambda_{0} t} / t$, where $\lambda_{0}$ is the lowest positive eigenvalue of the relevant linearized kinetics.

In Sec. IV, the linearized phonon-assisted kinetics of statistically-degenerate QW excitons is formulated and analyzed. We show that the eigenvalues $\{\lambda\}$ of the linear collision integral form a continuous spectrum, $\infty>$ $\lambda \geq \lambda_{0}>0$, separated from the nondegenerate eigenvalue $\lambda=0$, and that the corresponding eigenfunctions $\left\{\psi_{\varepsilon}(\lambda)\right\}$ have three well-defined isolated critical points. The dependence of the marginal eigenvalue $\lambda_{0}$ on the bath $\left(T_{b}\right)$ and degeneracy $\left(T_{0}\right)$ temperatures is studied.

In Sec. V, straightforward numerical simulations of the phonon-assisted relaxation kinetics at $T_{b} \lesssim T_{0}$ are compared with the generic solution of the quantum Boltzmann equation. We also show that at the beginning of the adiabatic stage of thermalization, at $t_{c} \leq t \lesssim \lambda_{0}^{-1}$, the population dynamics of the ground-state mode is given by $N_{\varepsilon=0}(t) \propto(1+\chi t)^{\nu}$, where the parameters $\chi$ and $\nu$ are calculated analytically. Furthermore, we propose a particular design of GaAs-based microcavities (MCs), which compromises the efficiency of LA-phonon-assisted scattering (the density of states is $\propto M_{x}$ ) with the degeneracy temperature $T_{0} \propto M_{x}^{-1}$, i.e., allows us to avoid the bottleneck effect in relaxation and, therefore, to optimize 
the thermalization kinetics of low-density QW excitons.

In the Appendix, some relationships relevant to the thermalization dynamics of quasi-equilibrated highenergy QW excitons are given.

\section{BOLTZMANN KINETIC EQUATION FOR DEGENERATE QW EXCITONS}

For hot QW excitons, which are in-plane isotropically distributed at $t=0$, the thermalization kinetics due to bulk LA-phonons can be treated in one-dimensional energy space [see Eq. (2) of Ref. 8]. In the following we express energy $E$ and temperature $T$ (as well as $T_{b}$ and
$\left.T_{0}\right)$ in terms of $E_{0}$, i.e., we use the dimensionless values of $E \rightarrow \varepsilon=E / E_{0}$, and $T \rightarrow\left(k_{B} T\right) / E_{0}$. In order to derive and analyze the generic solution for relaxation at $T_{b}<T_{0}$, it is convenient to rewrite the above equation in terms of the variable

$$
f_{\varepsilon}(t)=\frac{N_{\varepsilon}(t)-N_{\varepsilon}^{0}}{T_{b}\left(N_{\varepsilon}^{0}\right)^{\prime}}
$$

where $N_{\varepsilon}(t)$ and $N_{\varepsilon}^{0}=1 /\left[e^{(\varepsilon-\mu) / T_{b}}-1\right]$ are the current and (final) equilibrium distribution functions of QW excitons, respectively, and the chemical potential $\mu$ is given by $\mu=T_{b} \ln \left(1-e^{-T_{0} / T_{b}}\right)$. In this case the kinetic equation reduces to

$$
\frac{\partial}{\partial t} f_{\varepsilon}(t)=-\frac{4}{\tau_{\mathrm{sc}}}\left[\int_{0}^{\theta_{\mathrm{S}}(\varepsilon)} F_{\mathrm{S}}\left(\varepsilon, \varepsilon_{1}\right) \mathcal{L}_{\mathrm{S}}\left(\varepsilon, \varepsilon_{1}, t\right) \varepsilon_{1} d \varepsilon_{1}+\int_{\theta_{\mathrm{AS}}(\varepsilon)}^{\infty} F_{\mathrm{AS}}\left(\varepsilon, \varepsilon_{1}\right) \mathcal{L}_{\mathrm{AS}}\left(\varepsilon, \varepsilon_{1}, t\right) \varepsilon_{1} d \varepsilon_{1}\right]
$$

where the Stokes (S) and anti-Stokes (AS) collision integrands are

$$
\begin{aligned}
\mathcal{L}_{\mathrm{S}}\left(\varepsilon, \varepsilon_{1}, t\right)= & {\left[f_{\varepsilon}(t)-f_{\varepsilon-\varepsilon_{1}}(t)\right]\left(1+n_{\varepsilon_{1}}^{\mathrm{ph}}+N_{\varepsilon-\varepsilon_{1}}^{0}\right) } \\
& +T_{b}\left(N_{\varepsilon-\varepsilon_{1}}^{0}\right)^{\prime} f_{\varepsilon}(t) f_{\varepsilon-\varepsilon_{1}}(t), \\
\mathcal{L}_{\mathrm{AS}}\left(\varepsilon, \varepsilon_{1}, t\right)= & {\left[f_{\varepsilon}(t)-f_{\varepsilon+\varepsilon_{1}}(t)\right]\left(n_{\varepsilon_{1}}^{\mathrm{ph}}-N_{\varepsilon+\varepsilon_{1}}^{0}\right) } \\
& -T_{b}\left(N_{\varepsilon+\varepsilon_{1}}^{0}\right)^{\prime} f_{\varepsilon}(t) f_{\varepsilon+\varepsilon_{1}}(t) .
\end{aligned}
$$

The distribution of thermal bulk phonons is given by the Planck formula, i.e., $n_{\varepsilon}^{\mathrm{ph}}=1 /\left(e^{\varepsilon / T_{b}}-1\right)$. The scattering time is defined by $\tau_{\text {sc }}=\left(\pi^{2} \hbar^{4} \rho\right) /\left(D_{x}^{2} M_{x}^{3} v_{s}\right)$. The func- tions $\theta_{\mathrm{S} / \mathrm{AS}}(\varepsilon)$, which determine the integration limits on the right-hand-side of Eq. (2.2), are

$$
\begin{aligned}
\theta_{\mathrm{S}}(\varepsilon) & = \begin{cases}0, & \varepsilon \leq 1 / 4 \\
2 \sqrt{\varepsilon}-1, & 1 / 4<\varepsilon \leq 1 \\
\varepsilon, & 1<\varepsilon,\end{cases} \\
\theta_{\mathrm{AS}}(\varepsilon) & = \begin{cases}1-2 \sqrt{\varepsilon}, & \varepsilon \leq 1 / 4 \\
0, & \varepsilon>1 / 4 .\end{cases}
\end{aligned}
$$

The functions $F_{\mathrm{S} / \mathrm{AS}}\left(\varepsilon, \varepsilon_{1}\right)$ in Eq. (2.2) are given by

$$
F_{\mathrm{S} / \mathrm{AS}}\left(\varepsilon, \varepsilon_{1}\right)=\int_{d_{\varepsilon, \varepsilon_{1}}^{\mathrm{S} / \mathrm{AS}}}^{u_{\varepsilon, \varepsilon_{1}}^{\mathrm{S} / \mathrm{AS}}}\left|F_{z}\left(a \varepsilon_{1} \alpha\right)\right|^{2}\left\{\left[\left(u_{\varepsilon, \varepsilon_{1}}^{\mathrm{S} / \mathrm{AS}}\right)^{2}-\alpha^{2}\right]\left[\alpha^{2}-\left(d_{\varepsilon, \varepsilon_{1}}^{\mathrm{S} / \mathrm{AS}}\right)^{2}\right]\right\}^{-1 / 2} d \alpha
$$

where $u_{\varepsilon, \varepsilon_{1}}^{\mathrm{S} / \mathrm{AS}}=\left[1-\left(\sqrt{\varepsilon}-\sqrt{\varepsilon \mp \varepsilon_{1}}\right)^{2} / \varepsilon_{1}^{2}\right]^{1 / 2}$ and $d_{\varepsilon, \varepsilon_{1}}^{\mathrm{S} / \mathrm{AS}}=$ $\left[1-\left(\sqrt{\varepsilon}+\sqrt{\varepsilon \mp \varepsilon_{1}}\right)^{2} / \varepsilon_{1}^{2}\right]^{1 / 2}$. The dimensionless parameter $a$ in the argument of the form-factor function $F_{z}$ is given by $a=\left(L_{z} M_{x} v_{s}\right) / \hbar$.

In order to estimate the contribution of the form-factor $F_{z}(\chi)$ to the spectral functions $F_{\mathrm{S} / \mathrm{AS}}\left(\varepsilon, \varepsilon_{1}\right)$ one can analyze Eq. (2.5) for $\varepsilon \rightarrow 0$. In this case only the spectral function $F_{\mathrm{AS}}\left(\varepsilon, \varepsilon_{1}\right)$ is relevant to the kinetic Eq. (2.2), and Eq. (2.5) yields

$$
F_{\mathrm{AS}}\left(0, \varepsilon_{1}\right)=\frac{\pi}{2} \sqrt{\frac{\varepsilon_{1}}{\varepsilon_{1}-1}}\left|F_{z}\left[a \sqrt{\varepsilon_{1}\left(\varepsilon_{1}-1\right)}\right]\right|^{2}
$$

For $\varepsilon_{1} \gg 1$, the spectral width $\Delta \varepsilon$ (FWHM) of $F_{\mathrm{AS}}\left(0, \varepsilon_{1}\right)$ is given by $\Delta \varepsilon \simeq 2.26 / a$ and determined solely by $F_{z}\left(a \varepsilon_{1}\right)$. For thickness $L_{z}=8 \mathrm{~nm}$ of GaAs QWs (see Ref. 15) one gets $a \simeq 0.054$ and, therefore, $\Delta \varepsilon \simeq 42$. The latter estimate can be rewritten in the dimensional energy units as $\Delta E=E_{0} \Delta \varepsilon=4.52 \hbar v_{s} / L_{z} \simeq 1.44 \mathrm{meV}$. Thus the spectral band of LA-phonons, which scatter a low-energy QW exciton, can be evaluated as $\Delta E \sim$ $v_{s} \Delta p_{z}$, where $\Delta p_{z} \sim \hbar / L_{z}$ is the uncertainty of the momentum in the $z$-direction due to the QW spatial confinement. The above estimates show that for relatively cold QW excitons with energies $\varepsilon \leq \Delta \varepsilon$ (the effective temperature $T^{\mathrm{eff}}=\Delta E / k_{B} \simeq 16.5 \mathrm{~K}$ for $L_{z}=8 \mathrm{~nm}$ ) the form- 
factor can be approximated by $F_{z}\left(a \varepsilon_{1} \alpha\right)=F_{z}(0)=1$. In this case the integral on the r.h.s. of Eq. (2.5) can be calculated explicitly:

$$
F_{\mathrm{S} / \mathrm{AS}}\left(\varepsilon, \varepsilon_{1}\right)=\frac{1}{d_{\varepsilon, \varepsilon_{1}}^{\mathrm{S} / \mathrm{AS}}} \Phi\left[\left(\frac{d_{\varepsilon, \varepsilon_{1}}^{\mathrm{S} / \mathrm{AS}}}{u_{\varepsilon, \varepsilon_{1}}^{\mathrm{S} / \mathrm{AS}}}\right)^{2}\right]
$$

where

$\Phi(\xi)= \begin{cases}-i\left\{F\left[\arcsin (\sqrt{\xi}), \frac{1}{\xi}\right]-K\left(\frac{1}{\xi}\right)\right\}, & 0 \leq \xi<1 \\ K\left(\frac{1}{\xi}\right), & \xi<0 .\end{cases}$

Here, $F(\phi, m)=\int_{0}^{\phi}\left[1-m \sin ^{2}(\theta)\right]^{-1 / 2} d \theta$ and $K(m)=$ $F(\pi / 2, m)$ are the elliptic and the complete elliptic integrals of the first kind, respectively.

\section{GENERIC SOLUTION}

In this Section the thermalization kinetics at $T_{b} \leq$ $T_{0}$ is described in terms of a generic solution, which weakly correlates with the initial distribution $N_{\varepsilon}(t=0)$. In particular, we derive a thermalization law for highenergy excitons and examine nonequilibrium distribution of low-energy QW excitons. We also specify a reference point for the generic solution, i.e., a set of parameters, which unambiguously determines the calculated evolution. Schematic picture of the relaxation kinetics in phase space is shown in Fig. 2. The generic solution is relevant to the times $t>t_{c}$, where $t_{c}$ is the start time of the adiabatic stage of evolution.

As was emphasized in Sec. I, the generic solution assumes a quasi-equilibrium distribution of high-energy excitons $(\varepsilon>1 / 4): N_{\varepsilon>1 / 4}(t)=1 /\left[e^{(\varepsilon-\tilde{\mu}(t)) / T(t)}-1\right]$. This quasi-equilibrium distribution is characterized by the effective time-dependent temperature $T(t)=T_{b}+\delta T(t)$ and chemical potential $\tilde{\mu}(t)$ whose time variations are supposed to be small, so that $\tilde{\mu}(t)$ can be taken the same as the chemical potential of final equilibrium distribution $\tilde{\mu}(t) \simeq \mu$. Indeed, for $T(t)<T_{0}$ the chemical potential is given by $\tilde{\mu}(t) \simeq-T(t) e^{-T_{0} / T(t)} \ll T(t)$ and, therefore, $|\delta \mu|=|\tilde{\mu}(t)-\mu| \ll \delta T(t)$. Since at the final stage of relaxation kinetics the effective temperature of high-energy excitons approaches the bath temperature $(\delta T(t) \rightarrow 0)$, starting from some moment in time one meets the condition $\delta T(t) \ll T_{b}$ (provided that $T_{b}>0$ ). Therefore we can linearize the quasi-equilibrium distribution function with respect to $\delta T(t)$. In this case one obtains

$$
f_{\varepsilon>1 / 4}(t)=-\left[\delta T(t) / T_{b}^{2}\right] \varepsilon .
$$

On the other hand, as we show below, a low-energy kernel $(0<\varepsilon \leq 1 / 4)$ of the distribution function characterizes the non-equilibrium QW excitons. With increasing time $t \geq t_{c}$, the low-energy kernel shrinks in energy space, and the ratio $\left|N_{\varepsilon \leq 1 / 4}-N_{\varepsilon \leq 1 / 4}^{0}\right| / N_{\varepsilon \leq 1 / 4}^{0}$ decreases approaching the limit when the linearized kinetics becomes valid.

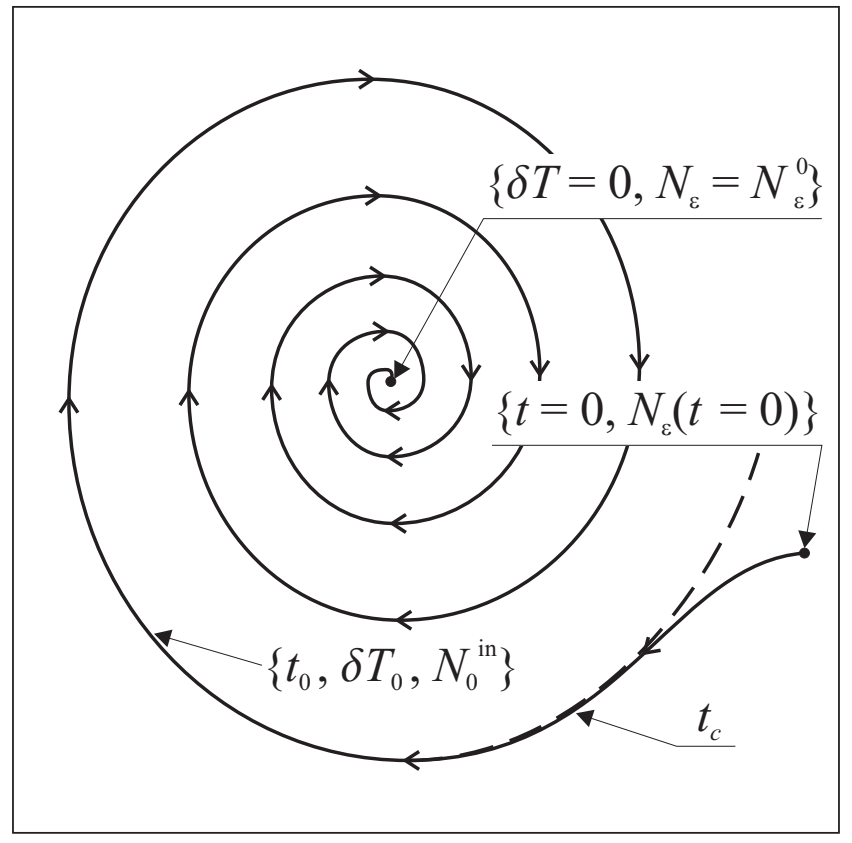

FIG. 2: Schematic picture of the relaxation kinetics at $T_{b} \lesssim T_{0}$. The reference (start) point $\left\{t_{0}, \delta T_{0}, N_{0}^{\text {in }}\right\}$ unambiguously determines the calculated (solid line) evolution from the initial noequilibrium distribution $N_{\varepsilon}(t=0)$. The dashed line corresponds to the generic solution. $t_{c}$ is the start point of adiabatic stage of evolution. For the times $t-t_{c} \geq \tau_{\mathrm{sc}}$ both evolution spirals almost coincide showing a unique path towards the final equilibrium distribution $\left\{\delta T=0, N_{\varepsilon}=N_{\varepsilon}^{0}\right\}$.

\section{A. Nonequilibrium distribution of low-energy QW excitons}

In order to analyze the evolution of low-energy QW excitons within the scenario described in the previous subsection, we substitute Eq. (3.1) into Eq. (2.2) and get the reduced kinetic equation for $\varepsilon \leq 1 / 4$ :

$$
\frac{\partial}{\partial t} f_{\varepsilon}(t)=-\left[\xi_{0}(\varepsilon)+\xi_{1}(\varepsilon) \delta T(t)\right] f_{\varepsilon}(t)+\eta(\varepsilon) \delta T(t),
$$

where

$$
\xi_{0}(\varepsilon)=\frac{4}{\tau_{\mathrm{sc}}} \int_{1-2 \sqrt{\varepsilon}}^{\infty} F_{\mathrm{AS}}\left(\varepsilon, \varepsilon_{1}\right)\left(n_{\varepsilon_{1}}^{\mathrm{ph}}-N_{\varepsilon+\varepsilon_{1}}^{0}\right) \varepsilon_{1} d \varepsilon_{1},
$$

$$
\xi_{1}(\varepsilon)=\frac{4}{\tau_{\mathrm{sc}} T_{b}} \int_{1-2 \sqrt{\varepsilon}}^{\infty} F_{\mathrm{AS}}\left(\varepsilon, \varepsilon_{1}\right)\left(N_{\varepsilon+\varepsilon_{1}}^{0}\right)^{\prime} \varepsilon_{1}\left(\varepsilon+\varepsilon_{1}\right) d \varepsilon_{1},
$$

$$
\begin{aligned}
\eta(\varepsilon)=-\frac{4}{\tau_{\mathrm{sc}} T_{b}^{2}} \int_{1-2 \sqrt{\varepsilon}}^{\infty} & F_{\mathrm{AS}}\left(\varepsilon, \varepsilon_{1}\right)\left(n_{\varepsilon_{1}}^{\mathrm{ph}}-N_{\varepsilon+\varepsilon_{1}}^{0}\right) \\
& \times \varepsilon_{1}\left(\varepsilon+\varepsilon_{1}\right) d \varepsilon_{1},
\end{aligned}
$$


and $F_{\mathrm{AS}}\left(\varepsilon+\varepsilon_{1}\right)$ is given by Eq. (2.7). Equation (3.2), which describes the population dynamics of the lowenergy states during the adiabatic stage of thermalization $\left(t \geq t_{c}\right)$, is a linear inhomogeneous differential equation for $f_{\varepsilon<1 / 4}$. Its complete solution can be written as a sum of the homogeneous and inhomogeneous contributions:

$$
\begin{aligned}
f_{\varepsilon \leq 1 / 4}(t)=f_{\varepsilon \leq 1 / 4}^{\text {hom }}(t)+\eta(\varepsilon) \int_{t_{0}}^{t} & e^{-(t-\tau) \xi_{0}(\varepsilon)-\rho(t, \tau) \xi_{1}(\varepsilon)} \\
& \times \delta T(\tau) d \tau
\end{aligned}
$$

where

$$
\begin{gathered}
f_{\varepsilon \leq 1 / 4}^{\mathrm{hom}}(t)=e^{-\left(t-t_{0}\right) \xi_{0}(\varepsilon)-\rho\left(t, t_{0}\right) \xi_{1}(\varepsilon)} f_{\varepsilon \leq 1 / 4}\left(t=t_{0}\right), \\
\rho\left(t, t_{1}\right)=\int_{t_{1}}^{t} \delta T(\tau) d \tau
\end{gathered}
$$

and $t_{0}$ is an arbitrary reference (start) time for the calculated evolution, i.e., $t_{0} \geq t_{c}$ (see Fig. 2).

Thus the adiabatic stage of the relaxation kinetics into the lower-energy states is completely determined by $N_{\varepsilon}(t)=N_{\varepsilon}^{0}+T_{b}\left(N_{\varepsilon}^{0}\right)^{\prime} f_{\varepsilon}(t)$, provided that one knows the reference (start) distribution $f_{\varepsilon \leq 1 / 4}\left(t=t_{0}\right)$ and thermalization law $\delta T=\delta T(t)$ for high-energy QW excitons. Note that in a sharp contrast with the acousticphonon-assisted relaxation kinetics at $T_{b} \leq T_{c}$ in threedimensional systems, 40 the thermalization dynamics of low-energy QW excitons depends upon the homogeneous contribution $f_{\varepsilon \leq 1 / 4}^{\text {hom }}$ given by Eq. (3.7).

As will be shown in the next subsection, the thermalization law for quasi-equilibrium high-energy QW excitons $(\varepsilon>1 / 4)$ is given by

$$
\delta T(t)=\left(\frac{\delta T_{0}}{\lambda_{1}-\lambda_{0}}\right) \frac{e^{-\lambda_{0}\left(t-t_{0}\right)}-e^{-\lambda_{1}\left(t-t_{0}\right)}}{t-t_{0}},
$$

where $\lambda_{0}=\xi_{0}(0)$ characterizes the inverse thermalization time at $t \rightarrow \infty, \lambda_{1}=\xi_{0}(1 / 4)$ is another characteristic parameter relevant to the beginning of the adiabatic stage $\left(\lambda_{1} \gg \lambda_{0}\right)$, and $\delta T_{0}=\delta T\left(t=t_{0}\right)$ determines reference (start) effective temperature $T\left(t=t_{0}\right)=T_{b}+\delta T_{0}$ for the calculated evolution. Equation (3.9) is valid for $t \geq t_{0}$. Using the thermalization law $(3.9)$ we find the integral on the r.h.s. of Eq. (3.8):

$$
\begin{aligned}
\rho\left(t, t_{1}\right)= & \frac{\delta T_{0}}{\lambda_{1}-\lambda_{0}}\left\{E i\left[-\lambda_{0}\left(t-t_{0}\right)\right]-E i\left[-\lambda_{0}\left(t_{1}-t_{0}\right)\right]\right. \\
& \left.-E i\left[-\lambda_{1}\left(t-t_{0}\right)\right]+E i\left[-\lambda_{1}\left(t_{1}-t_{0}\right)\right]\right\},(3.10)
\end{aligned}
$$

where $\operatorname{Ei}(z)=-\int_{-z}^{\infty}\left(e^{-t} / t\right) d t$ is the exponential integral function.

The reference distribution $f_{\varepsilon \leq 1 / 4}\left(t=t_{0}\right)$, which determines the homogeneous solution (3.7), is independent of the initial distribution of hot QW excitons, $N_{\varepsilon}(t=0)$. There is, however, an integral relationship between $\delta T_{0}$ and $f_{\varepsilon \leq 1 / 4}\left(t=t_{0}\right)$, which we will discuss in subsection III along with possible approximations for $f_{\varepsilon \leq 1 / 4}(t=$ $\left.t_{0}\right)$.

\section{B. Thermalization of high-energy QW excitons}

The temperature change $\delta T=\delta T(t)$ characterizes the time evolution of high-energy quasi-equilibrated particles through Eqs. (2.1) and (3.1). In order to derive the temperature law (3.9) we substitute $f_{\varepsilon \leq 1 / 4}$ and $f_{\varepsilon>1 / 4}$ given by Eq. (3.6) and (3.1), respectively, into the kinetic equation (2.2):

$$
\frac{\partial}{\partial t} \delta T(t)=-\left[\alpha_{0}+\alpha_{1}(t)\right] \delta T(t)+\beta \delta T^{2}(t)+\gamma(t),
$$

where

$$
\begin{aligned}
\gamma(t)=-\frac{4 T_{b}^{2}}{\tau_{\mathrm{sc}} \varepsilon_{c}} \int_{0}^{1 / 4} & F_{\mathrm{S}}\left(\varepsilon_{c}, \varepsilon_{c}-\varepsilon\right)\left(1+n_{\varepsilon_{c}-\varepsilon}^{\mathrm{ph}} N_{\varepsilon}^{0}\right) \\
& \times f_{\varepsilon}(t)\left(\varepsilon_{c}-\varepsilon\right) d \varepsilon,
\end{aligned}
$$

and the parameters $\alpha_{0}, \beta$, and functional $\alpha_{1}(t)=$ $\alpha_{1}\left[f_{\varepsilon \leq 1 / 4}(t)\right]$ are defined in Appendix. Equations (3.11)(3.12) refer to some energy $\varepsilon_{c} \gtrsim 1$ from the high-energy domain $\varepsilon>1 / 4$.

In the adiabatic stage of relaxation, when $t>t_{c}$, one has $\delta T(t) / T_{b} \ll 1$ so that $\alpha_{1}$ and $\beta \delta T^{2}$ can be neglected on the r.h.s. of Eq. (3.11), because $\left|\alpha_{1}(t)\right| \ll \alpha_{0}$ and $\left|\beta \delta T^{2}(t)\right| \ll|\gamma(t)|$. At the end of the adiabatic stage $\left(t-t_{c} \gg \lambda_{0}^{-1}\right)$ the distribution function $N_{\varepsilon}(t)$ is already very close to $N_{\varepsilon}^{0}$ even for small energies $\varepsilon \leq 1 / 4$ and, therefore, the phonon-assisted relaxation kinetics becomes exponential, i.e., $\delta T(t) \propto e^{-\lambda_{0} t}$. For statisticallydegenerate QW excitons $\left(N_{\varepsilon=0}^{0} \gg 1\right)$ one estimates from Eqs. (3.3) and (A1) that positive $\lambda_{0}=\xi(0)$ is much less than positive $\alpha_{0}$. In this case Eq. (3.11) can be solved iteratively. The first iteration, which can formally be obtained by neglecting the time derivative on the r.h.s. of Eq. (3.11, yields

$$
\delta T(t)=\gamma(t) / \alpha_{0}
$$

For the same time domain $t-t_{c} \gg \lambda_{0}^{-1} \mathrm{Eq}$. (3.6) yields the following approximation:

$$
f_{\varepsilon \leq 1 / 4}(t)=e^{-\left(t-t_{0}\right) \xi_{0}(\varepsilon)} f_{\varepsilon}\left(t=t_{0}\right)+\frac{\eta(\varepsilon)}{\xi_{0}(\varepsilon)} \delta T(t) .
$$

By substituting Eq. (3.14) into the r.h.s. of Eq. (3.12) one derives from Eq. (3.13):

$$
\begin{aligned}
\delta T(t)=\frac{1}{\tilde{\alpha}} \int_{0}^{1 / 4} & F_{\mathrm{S}}\left(\varepsilon_{c}, \varepsilon_{c}-\varepsilon\right)\left(1+n_{\varepsilon_{c}-\varepsilon}^{\mathrm{ph}} N_{\varepsilon}^{0}\right) f_{\varepsilon}\left(t_{0}\right) \\
& \times e^{-\left(t-t_{0}\right) \xi_{0}(\varepsilon)}\left(\varepsilon_{c}-\varepsilon\right) d \varepsilon,
\end{aligned}
$$

where the constant $\tilde{\alpha}$ is defined in Appendix. We can further simplify Eq. (3.15) taking into account that $\xi_{0}(\varepsilon)$ is a monotonously increasing function of energy and that at large $t$ only small vicinity of $\varepsilon=0$ contributes to the integral. Finally, we end up with the asymptotic law:

$$
\delta T(t)=\left(\frac{\tilde{\gamma}_{0}}{\tilde{\alpha}}\right) \frac{e^{-\xi_{0}(0)\left(t-t_{0}\right)}-e^{-\xi_{0}(1 / 4)\left(t-t_{0}\right)}}{t-t_{0}},
$$




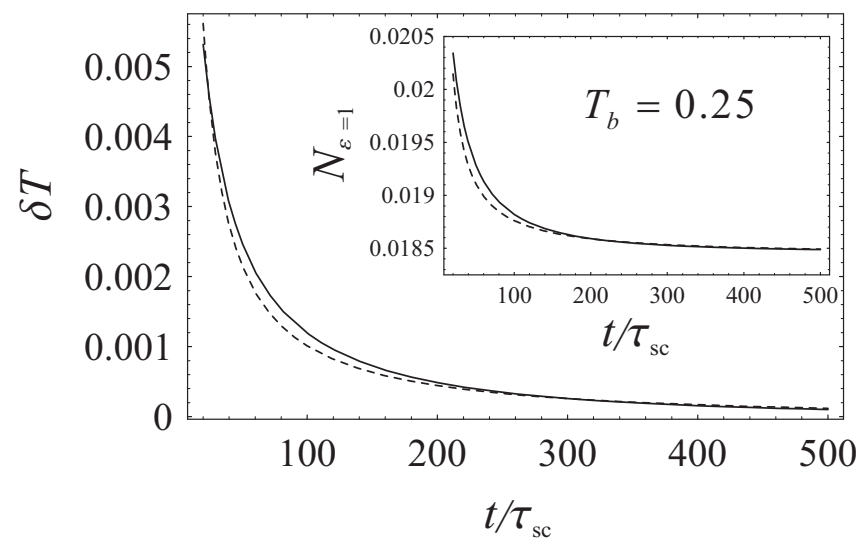

FIG. 3: Time dependence of $\delta T$ and $N_{\varepsilon=1}$ (inset) calculated by using the thermalization law (3.9) (dashed lines) and by direct numerical modelling of the initial kinetic Eq. (2.2) (solid lines). The control parameters $T_{b}$ and $T_{0}$ are the same as in Fig. 11.

where the constant $\tilde{\gamma}_{0}$ is given in Appendix. Equation (3.16) is identical to Eq. (3.9) provided that the reference (start) temperature $T_{b}+\delta T\left(t=t_{0}\right)$ of the high-energy quasi-equilibrated QW excitons is determined by

$$
\delta T_{0} \equiv \delta T\left(t=t_{0}\right)=\left(\tilde{\gamma}_{0} / \tilde{\alpha}\right)\left(\lambda_{1}-\lambda_{0}\right)
$$

While the above derivation of Eqs. (3.13)-(3.17) assumes that $t-t_{c} \geq t-t_{0} \gg \lambda_{0}^{-1}$, we have checked numerically that the temperature law (3.9) holds through the whole adiabatic stage, i.e., for the time interval $0 \leq$ $t-t_{0}<\infty$. Furthermore, the numerical evaluations also clearly indicate that the start temperature $\delta T\left(t=t_{0}\right)$ is practically independent of the local energy $\varepsilon_{c} \gtrsim 1$ used in the derivation of Eqs. (3.11)-(3.17). In Fig. 3 we plot $\delta T=\delta T(t)$ and $N_{\varepsilon=1}=N_{\varepsilon=1}(t)$ calculated by using the thermalization law (3.9) (dashed lines) and by direct numerical modelling of the initial kinetic Eq. (2.2) (solid lines), respectively.

For the time domain $\lambda_{1}^{-1} \leq t-t_{0} \lesssim \lambda_{0}^{-1}$ the thermalization law (3.9) can be approximated by $\delta T(t)=\left(\lambda_{1}-\right.$ $\left.\lambda_{0}\right)^{-1} \delta T_{0} /\left(t-t_{0}\right)$. The latter dependence $\delta T(t) \propto 1 /(t-$ $\left.t_{0}\right)$ is consistent with that found for the adiabatic stage of the phonon-assisted relaxation kinetics of 3D bosons (excitons) at $T_{b} \leq T_{c}$, when Bose-Einstein condensate builds up. 40 For $2 \mathrm{D}$ systems at nonzero $T_{b}$ the occupation number of the ground-state mode $N_{\varepsilon=0}^{0}$ is always final. Therefore the exponential kinetics $\delta T(t) \propto e^{-\lambda_{0}\left(t-t_{0}\right)}$, which results from Eq. (3.9) for $t-t_{0} \gtrsim \lambda_{0}^{-1}$, develops at the final stage of relaxation, when $\left|N_{\varepsilon=0}(t)-N_{\varepsilon=0}^{0}\right| / N_{\varepsilon=0}^{0} \ll 1$. Note that because for statistically-degenerate QW excitons, when $T_{b} \lesssim T_{0}$ and $N_{\varepsilon=0}^{0} \gg 1$, one has $\lambda_{0}^{-1} \gg \tau_{s c}$ (see Section IV), the two modes of behavior, $\delta T \propto 1 / t$ and $\delta T \propto e^{-\lambda_{0} t}$, are well-separated in time.

\section{Reference point for the generic solution}

In order to determine the relaxation kinetics of lowenergy particles, i.e., $N_{\varepsilon \leq 1 / 4}=N_{\varepsilon \leq 1 / 4}\left(t \geq t_{c}\right)$, one needs to specify the reference distribution $f_{\varepsilon \leq 1 / 4}\left(t=t_{0}\right)$ and $\delta T_{0}=\delta T\left(t=t_{0}\right)=T\left(t=t_{0}\right)-T_{b}$. According to Eq. (3.7), with increasing time $t-t_{0}$ only the small vicinity of $\varepsilon=0$ gives contribution to the homogeneous part of the generic solution. Therefore we approximate $N_{\varepsilon \leq 1 / 4}\left(t=t_{0}\right) \simeq N_{0}^{\text {in }} \equiv N_{\varepsilon=0}\left(t=t_{0}\right)$. The above approximation assumes that the reference time $t_{0} \geq t_{c}$ is close enough to the start time $t_{c}$ of the adiabatic stage of relaxation so that $N_{\varepsilon}\left(t_{0}\right) \ll N_{\varepsilon}^{0}$ at $\varepsilon \ll 1 / 4$. Thus, using Eq. (2.1) we determine the reference distribution at $t=t_{0}$ by

$$
f_{\varepsilon \leq 1 / 4}\left(t=t_{0}\right)=\frac{N_{0}^{\mathrm{in}}-N_{\varepsilon}^{0}}{T_{b}\left(N_{\varepsilon}^{0}\right)^{\prime}} .
$$

The latter expression is completely defined by the only one unknown parameter $N_{0}^{\text {in }}$, the population of the ground-state mode at the reference time $t_{0}$.

Equation (3.18) allows us to find $\delta T_{0}=\delta T\left(t=t_{0}\right)$ through the integral relationship (3.15) taken at $t=t_{0}$. Furthermore, within the approximations used in the derivation of the asymptotic law (3.16) a simplified (algebraic) form of this relationship, given by Eqs. (3.17) and (A5), is valid as well. After the value of $\delta T_{0}$ is determined, using the thermalization law (3.9) one can easily find the time dependence of the distribution function (3.1) of high-energy excitons. Therefore, the three parameters $t_{0}, N_{0}^{\text {in }}$, and $\delta T_{0}$, completely specify the reference point for the generic solution, as illustrated in Fig. 2. 2 .

\section{LINEARIZED KINETICS FOR STATISTICALLY DEGENERATE QW EXCITONS}

If for any energy $\varepsilon$ the distribution function $N_{\varepsilon}$ of quantum-degenerate quasi-2D excitons is close enough to final $N_{\varepsilon}^{0}$ so that $\left|N_{\varepsilon}-N_{\varepsilon}^{0}\right| / N_{\varepsilon}^{0} \ll 1$ and $f_{\varepsilon}(t)$ becomes small, the phonon-assisted kinetics can be linearized. In particular, the adiabatic stage of thermalization at times $t-t_{0} \geq \lambda_{0}^{-1}$ refers to the linearized kinetics. The linearized kinetics can be described in terms of the real eigenvalues $\{\lambda\}(\lambda \geq 0)$ and the corresponding eigenfunctions $\left\{\psi_{\varepsilon}(\lambda)\right\}$ so that $f_{\varepsilon}(t)=\sum_{\lambda} c_{\lambda} \psi_{\varepsilon}(\lambda) \exp (-\lambda t)$. The initial kinetic Eq. (2.2) reduces to the linear Fredholm equation of the second kind with respect to $\psi_{\varepsilon}(\lambda)$ :

$$
\begin{aligned}
\lambda \psi_{\varepsilon}(\lambda)= & \frac{4}{\tau_{\mathrm{sc}}}\left[\int_{0}^{\theta_{\mathrm{S}}(\varepsilon)} F_{\mathrm{S}}\left(\varepsilon, \varepsilon_{1}\right) \tilde{\mathcal{L}}_{\mathrm{S}}\left(\varepsilon, \varepsilon_{1}\right) \varepsilon_{1} d \varepsilon_{1}\right. \\
& \left.+\int_{\theta_{\mathrm{AS}}(\varepsilon)}^{\infty} F_{\mathrm{AS}}\left(\varepsilon, \varepsilon_{1}\right) \tilde{\mathcal{L}}_{\mathrm{AS}}\left(\varepsilon, \varepsilon_{1}\right) \varepsilon_{1} d \varepsilon_{1}\right]
\end{aligned}
$$

where

$$
\tilde{\mathcal{L}}_{\mathrm{S}}\left(\varepsilon, \varepsilon_{1}\right)=\left[\psi_{\varepsilon}(\lambda)-\psi_{\varepsilon-\varepsilon_{1}}(\lambda)\right]\left(1+n_{\varepsilon_{1}}^{\mathrm{ph}}+N_{\varepsilon-\varepsilon_{1}}^{0}\right),
$$




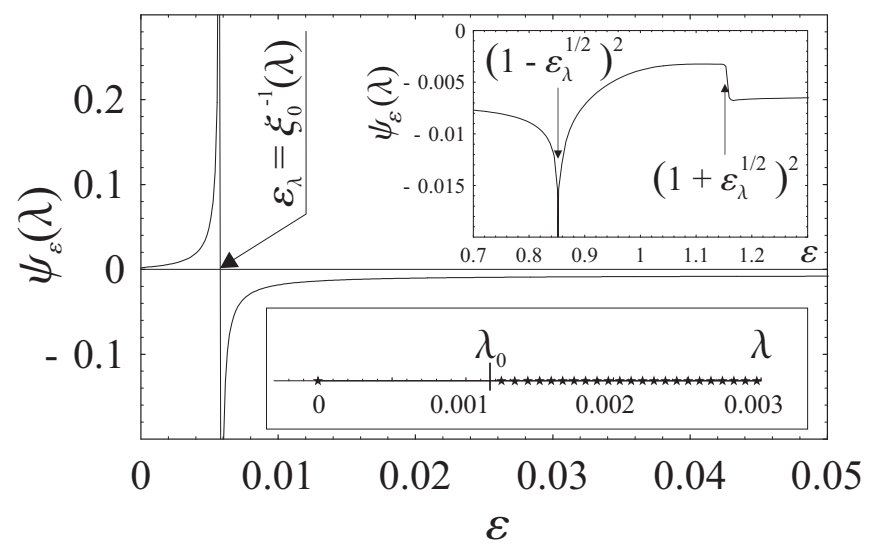

FIG. 4: A typical shape of the eigenfunction $\psi_{\varepsilon}(\lambda)$. The main part of the figure shows the first order pole, which arises at the energy region $\varepsilon<1 / 4\left(\lambda=0.0043 / \tau_{\text {sc }}\right.$ in this particular example). The top inset illustrates eigenfunction behavior at critical points in the high energy band $\varepsilon \geq 1$. The eigenvalue spectrum ( $\lambda$ in units $\left.1 / \tau_{\text {sc }}\right)$ is shown in the bottom inset. The control parameters $T_{b}$ and $T_{0}$ are the same as in Fig. 1.

$$
\tilde{\mathcal{L}}_{\mathrm{AS}}\left(\varepsilon, \varepsilon_{1}\right)=\left[\psi_{\varepsilon}(\lambda)-\psi_{\varepsilon+\varepsilon_{1}}(\lambda)\right]\left(n_{\varepsilon_{1}}^{\mathrm{ph}}-N_{\varepsilon+\varepsilon_{1}}^{0}\right),
$$

and functions $\theta_{\mathrm{S}}$ and $\theta_{\mathrm{AS}}$ are given by Eqs. (2.4a) and (2.4b), respectively.

Thus we replace the solution of Eq. (2.2) by the eigenfunction analysis of the Fredholm integral equation (4.1). The numerical solution of Eq. (4.1) clearly shows that for a given $T_{b}>0$ all except one eigenvalues $\{\lambda\}$ are nondegenerate, positive, and belong to the continuous spectrum. This is illustrated in the bottom inset of Fig. A, where the set of eigenvalues shown by the stars covers the same interval $\infty>\lambda \geq \lambda_{0}=\lambda_{0}\left(T_{b}, T_{0}\right)$ more dense the more discrete points in energy space are used. The isolated non-degenerate eigenvalue $\lambda=0$ is due to conservation of the total number of QW excitons in our model (the only integral of motion of the system 11 ).

Since all $\{\lambda\}$ are nondegenerate, the corresponding eigenfunctions $\left\{\psi_{\varepsilon}(\lambda)\right\}$ form a basis in Hilbert energyspace. For the energy band $0 \leq \varepsilon<1 / 4$ one derives from Eq. (4.1):

$$
\psi_{\varepsilon<1 / 4}(\lambda)=\frac{\sigma(\varepsilon)}{\lambda-\xi_{0}(\varepsilon)},
$$

where $\sigma(\varepsilon)$ is a smooth regular function of $\varepsilon$ given by

$$
\begin{aligned}
\sigma(\varepsilon)=-\frac{4}{\tau_{\mathrm{sc}}} \int_{0}^{\theta_{\mathrm{S}}(\varepsilon)} & F_{\mathrm{S}}\left(\varepsilon, \varepsilon_{1}\right) \psi_{\varepsilon+\varepsilon_{1}}(\lambda) \\
& \times\left(n_{\varepsilon_{1}}^{\mathrm{ph}}-N_{\varepsilon+\varepsilon_{1}}^{0}\right) \varepsilon_{1} d \varepsilon_{1},
\end{aligned}
$$

and $\xi_{0}(\varepsilon)$ is defined by Eq. (3.3). Thus the eigenfunction $\psi_{\varepsilon}(\lambda)$ has an isolated singularity (first order pole) at $\varepsilon_{\lambda}=\xi_{0}^{-1}(\lambda)$. The singularity is integrable in terms of principal value integration. A typical shape of the eigenfunctions at $\varepsilon<1 / 4$ is shown in Fig. 田. In the energy band $\varepsilon \geq 1 / 4$ the eigenfunction has another singularity

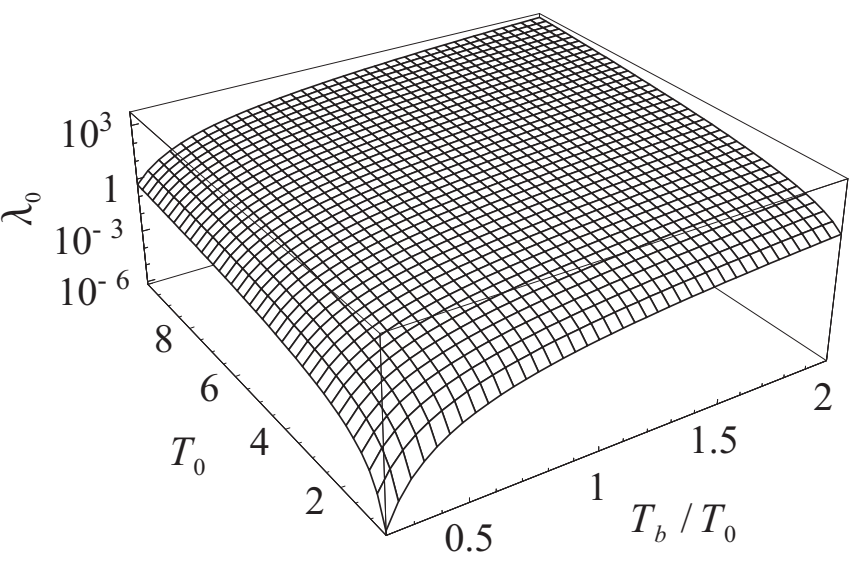

FIG. 5: The inverse thermalization time $\lambda_{0}$ (in units $1 / \tau_{\mathrm{sc}}$ ) as a function of the control parameters $T_{b}$ and $T_{0}$.

at the point $\left(1-\sqrt{\varepsilon_{\lambda}}\right)^{2}$. This singularity is logarithmic, i.e., integrable. It arises when the singularity of $\psi_{\varepsilon-\varepsilon_{1}}(\lambda)$ at $\varepsilon-\varepsilon_{1}=\varepsilon_{\lambda}$ [see Eq. (4.2)] coincides with the upper boundary of integration, $\overline{\theta_{\mathrm{S}}}(\varepsilon)$, in the Stokes collision term on the r.h.s. of Eq. (4.1). The step-like jump at the critical point $\left(1+\sqrt{\varepsilon_{\lambda}}\right)^{2}$ does not accompanied by discontinuity of the eigenfunction. The latter critical point originates from a singular behavior of the form-factor function $F_{\mathrm{S}}\left(\varepsilon, \varepsilon_{1}\right)$ at $\varepsilon_{1}=2 \sqrt{\varepsilon}-1$. The features of the eigenfunction $\psi_{\varepsilon}(\lambda)$ at points $\varepsilon=\left(1 \pm \sqrt{\varepsilon_{\lambda}}\right)^{2}$ are shown in the top inset of Fig. 1 .

The marginal point $\lambda_{0}=\lambda_{0}\left(T_{b}, T_{0}\right)$ of the continuous spectrum of $\{\lambda\}$ is indeed given by $\lambda_{0}=\xi_{0}(0)$ : for $\lambda \rightarrow \lambda_{0}$ the singularity point $\varepsilon_{\lambda} \rightarrow 0$, i.e., approaches its lowest limit. Because $\lambda_{0}^{-1}$ is the longest relaxation time generated by the continuum $\lambda_{0} \leq \lambda<\infty$, the eigenavalue $\lambda_{0}$ determines the phonon-assisted kinetics at $t \rightarrow \infty$ and, in particular, yields the characteristic thermalization time in the relaxation thermodynamics developed in Ref. 8. The dependence of $\lambda_{0}$ upon the control parameters of the system, $T_{b}$ and $T_{0}$, is plotted in the Fig. 5 .

The eigenvalue $\lambda_{0}$ can reach both limits, i.e., $\lambda_{0} \gg \tau_{\mathrm{sc}}^{-1}$ and $\lambda_{0} \ll \tau_{\text {sc }}^{-1}$ (see Fig. 5). The first limit, which corresponds to the considerable acceleration of the thermalization kinetics in comparison with that in the $3 \mathrm{D}$ case, is due to the relaxation of momentum conservation in $\mathrm{QW}$ exciton - bulk acoustic phonon scattering. The slowing down of thermalization, $\lambda_{0} \ll \tau_{\mathrm{sc}}^{-1}$, occurs (i) at low bath temperatures $T_{b} \leq 1$, due to the exponentially decreasing number of thermal acoustic phonons with energy $\varepsilon \geq 1$, and/or (ii) for well-developed quantum statistics $\left(T_{0} \gg\right.$ $T_{b}$ ), due to an effective suppression of the stimulated kinetic processes. There is no direct phonon-mediated interaction of low-energy QW excitons $\varepsilon \leq 1 / 4$, and at low temperatures $T_{b} \leq 1$ the relaxation kinetics occurs by the two-step process: "low-energy QW exciton $(\varepsilon \ll 1 / 4)$ + phonon $\left(\varepsilon_{1} \simeq 1\right) \rightarrow$ QW exciton $\left(\varepsilon_{2}=\varepsilon+\varepsilon_{1} \simeq 1\right)$ $\rightarrow$ low-energy QW exciton $\left(\varepsilon_{4}=\varepsilon+\varepsilon_{1}-\varepsilon_{3} \ll 1 / 4\right)$ + phonon $\left(\varepsilon_{3} \simeq 1\right)$ ". The first, anti-Stokes transition quenches with decreasing temperature $T_{b} \leq 1$ and yields 
a temperature dependent bottleneck effect in thermalization. In turn, the critical slowing down of the relaxation kinetics at $T_{0} \gg T_{b}$ arises due to mutual compensation of two stimulated fluxes, into and out the lowenergy QW states $\varepsilon \ll 1$. For example, for the groundstate mode $\varepsilon=0$ the collision integrand responsible for the stimulated kinetics is given by $N_{\varepsilon=0}\left(N_{\varepsilon_{1} \geq 1}-n_{\varepsilon_{1}>1}^{\mathrm{ph}}\right)$. At $T_{0} \gg T_{b}$ the latter combination becomes small in spite of a large occupation number $N_{\varepsilon=0} \gg 1$, because $\left|N_{\varepsilon_{1} \geq 1}-n_{\varepsilon_{1} \geq 1}^{\mathrm{ph}}\right| \simeq\left|N_{\varepsilon_{1} \geq 1}^{0}-n_{\varepsilon_{1} \geq 1}^{\mathrm{ph}}\right| \rightarrow 0$ as a result of a very small value of the chemical potential, $|\mu| \ll 1$.

Now we can give an alternative prove of the temperature law (3.9) which refers to high-energy QW excitons with $\varepsilon>1 / 4$. Namely, the function $f_{\varepsilon}\left(t=t_{0}\right)$ can be expanded over the basis $\left\{\psi_{\varepsilon}(\lambda)\right\}$. Then the solution of Eq. (2.2) is given by

$$
f_{\varepsilon}(t)=\int_{\lambda_{0}}^{\infty} c_{\lambda} \psi_{\varepsilon}(\lambda) e^{-\lambda\left(t-t_{0}\right)} d \lambda
$$

where $c_{\lambda}$ are the expansion coefficients. At large times $t-t_{0} \gg \tau_{\text {th }}$ only a small vicinity of $\lambda$ near $\lambda_{0}$ contributes to the integral, due to the time exponent in the integrand. If now one assumes a regular distribution of quasi-2D excitons at $t=t_{0}$, the coefficients $c_{\lambda}$ smoothly depend upon $\lambda$. As a result, the approximation $c_{\lambda} \simeq c_{\lambda_{0}}$ can be used in the integrand on the r.h.s. of Eq. (4.5). Furthermore, the eigenfunctions $\psi_{\varepsilon}(\lambda)$ have nearly the same smooth shape at high energies, as illustrated by Fig. 5. Thus we can also put $\psi_{\varepsilon}(\lambda) \simeq \psi_{\varepsilon}\left(\lambda_{0}\right)$ on the r.h.s. of Eq. (4.5). As a result, both $c_{\lambda}$ and $\psi_{\varepsilon}(\lambda)$ can be extracted out the integral. Using Eq. (3.1) we immediately get $\delta T(t) \propto e^{-\lambda_{0}\left(t-t_{0}\right)} /\left(t-t_{0}\right)$, which coincides with Eq. (3.9) at $t-t_{0} \gg \lambda_{1}^{-1}$. Note that the above derivation is based on the particular spectrum $(\lambda=0+$ continuum $\lambda_{0} \leq \lambda<\infty$ ) of the linearized collision integral and has no analogy in the relaxation kinetics due to particleparticle interaction. In the latter case the fivefold degenerate eigenvalue $\lambda=0$ is separated from the continuous spectrum by a set of discrete isolated eigenvalues. 41

\section{DISCUSSION}

In order to test the generic solution (3.3)- 3.10 ) we model the phonon-assisted relaxation of excitons within the initial kinetic Eq. (1.1) reduced to energy space [Eqs. (2.1)-(2.2)]. An adaptive inhomogeneous grid with 100 - 200 points for $\varepsilon$ is used to cover the close vicinity of the ground-state mode $\varepsilon=0$ (the maximum value of the dimensionless energy is $\varepsilon_{\max }=20$ ). Equation (2.2) is evaluated by a fourth-order Runge-Kutta integration routine with the time step $0.001-0.01 \tau_{\mathrm{sc}}$. In order to calculate integrals on the r.h.s. of Eq. (2.2) we perform spline-approximation for $f_{\varepsilon}(t)$ at every iterative step.

In numerical simulations we use the dimensionless temperatures $T_{b}$ and $T_{0}$ and measure time in $\tau_{\mathrm{sc}}$. This makes our results suitable for various QWs and sets of the control parameters, provided that $E_{0}$, and $\tau_{\mathrm{sc}}$ are specified. In Fig. 6 time evolution of the distribution $N_{\varepsilon}(t)=N_{\varepsilon}^{0}+T_{b}\left(N_{\varepsilon}^{0}\right)^{\prime} f_{\varepsilon}(t)$ as a numerical solution of Eq. (2.2) is compared with the corresponding generic solution (3.3)-(3.10) relevant to $T_{b}<T_{0}$. All plots demonstrate an excellent agreement between analytical and numerical solutions. Note, that at high $T_{b}$, e.g., $T_{b}=10$ (see the top plot in Fig. 6), the thermalization time $\tau_{\text {th }}=\lambda_{0}^{-1}$ achieves the limit $\tau_{\text {th }} \ll \tau_{\text {sc }}$, and the relative duration of the adiabatic stage estimated in terms of $t_{c}$ (duration of the first transient) becomes smaller than that at $T_{b} \lesssim 1$. In this case the influence of the initial distribution $N_{\varepsilon}(t=0)$ slightly affects the calculated evolution at the beginning of the adiabatic stage, and the analytical solution fits numerically simulated data a little worse as can be seen for the distribution functions at $t=0.015 \tau_{\mathrm{sc}}$. In contrast, at low $T_{b}$ the relaxation kinetics at the adiabatic stage is slow. For example, at $T_{b} \leq 0.25$ it lasts more than 1000 scattering times, where typical values of $\tau_{\mathrm{sc}}$ in GaAs/AlGaAs coupled QWs are on a scale of tens nanoseconds.

In Fig. 7 we also compare time dependences for the ground-state mode population $N_{\varepsilon=0}=N_{\varepsilon=0}(t)$ obtained numerically (solid lines) and analytically (dashed lines). Again, this figure shows that at $\varepsilon=0$ the generic solution (3.3)-(3.10) reproduces the adiabatic stage of the phononassisted relaxation kinetics very well. Within the time interval $0 \leq t-t_{0} \lesssim \tau_{\text {th }}$ the generic solution yields the following simple approximation for the adiabatic kinetics into the ground state mode:

$$
N_{\varepsilon=0}(t)=N_{0}^{\text {in }}\left[1+\chi\left(t-t_{0}\right)\right]^{\nu} .
$$

Parameters $\chi$ and $\nu$ can be found comparing series expansions of Eq. (5.1) and (3.6) about the point $t=t_{0}$. In such a way we get $\chi=\left(c_{1}^{2}-c_{2} N_{0}^{\text {in }}\right) /\left(c_{1} N_{0}^{\text {in }}\right)$ and $\nu=c_{1}^{2} /\left(c_{1}^{2}-c_{2} N_{0}^{\text {in }}\right)$, where time-independent constants $c_{1}$ and $c_{2}$ are given by

$$
\begin{aligned}
c_{1}= & \left(N_{\varepsilon=0}^{0}-N_{0}^{\mathrm{in}}\right)\left[\lambda_{0}+\delta T_{0} \xi_{1}(0)\right]+T_{b} \delta T_{0} \eta(0)\left(N_{\varepsilon=0}^{0}\right)^{\prime}, \\
c_{2}= & \frac{1}{2}\left(\left(N_{0}^{\mathrm{in}}-N_{\varepsilon=0}^{0}\right)\right. \\
& \times\left\{2 \lambda_{0}^{2}+\delta T_{0} \xi_{1}(0)\left[5 \lambda_{0}+\lambda_{1}+2 \delta T_{0} \xi_{1}(0)\right]\right\} \\
& \left.-T_{b} \delta T_{0} \eta(0)\left(N_{\varepsilon=0}^{0}\right)^{\prime}\left[3 \lambda_{0}+\lambda_{1}+2 \delta T_{0} \xi_{1}(0)\right]\right) . \quad
\end{aligned}
$$

Time dependences of the ground-state mode population $N_{\varepsilon=0}(t)$ calculated at $T_{b}=1$ and $T_{b}=2$ using Eq. (5.1) are shown in Fig. [7(a) with dash-dotted lines. At $t-t_{0}>$ $\tau_{\text {th }}$ the approximation (5.1) violates because the phononassisted relaxation kinetics starts to become exponential.

As we have shown in Sec. IV, for a given concentration of QW excitons $\rho_{2 \mathrm{D}} \lesssim 10^{9} \mathrm{~cm}^{-2}$ the thermalization kinetics slows down with decreasing $T_{b} / T_{0}$, i.e., with development of quantum statistics. By increasing both temperatures, $T_{0}$ and $T_{b}$, and keeping unchanged the ratio $T_{0} / T_{b} \gg 1$ one can simultaneously avoid the above bottleneck effect in relaxation and achieve high population of the ground-state mode, $N_{\varepsilon=0} \gg 1$. However, in this case the concentration of excitons $\rho_{2 \mathrm{D}} \propto T_{0}$ increases as well, 


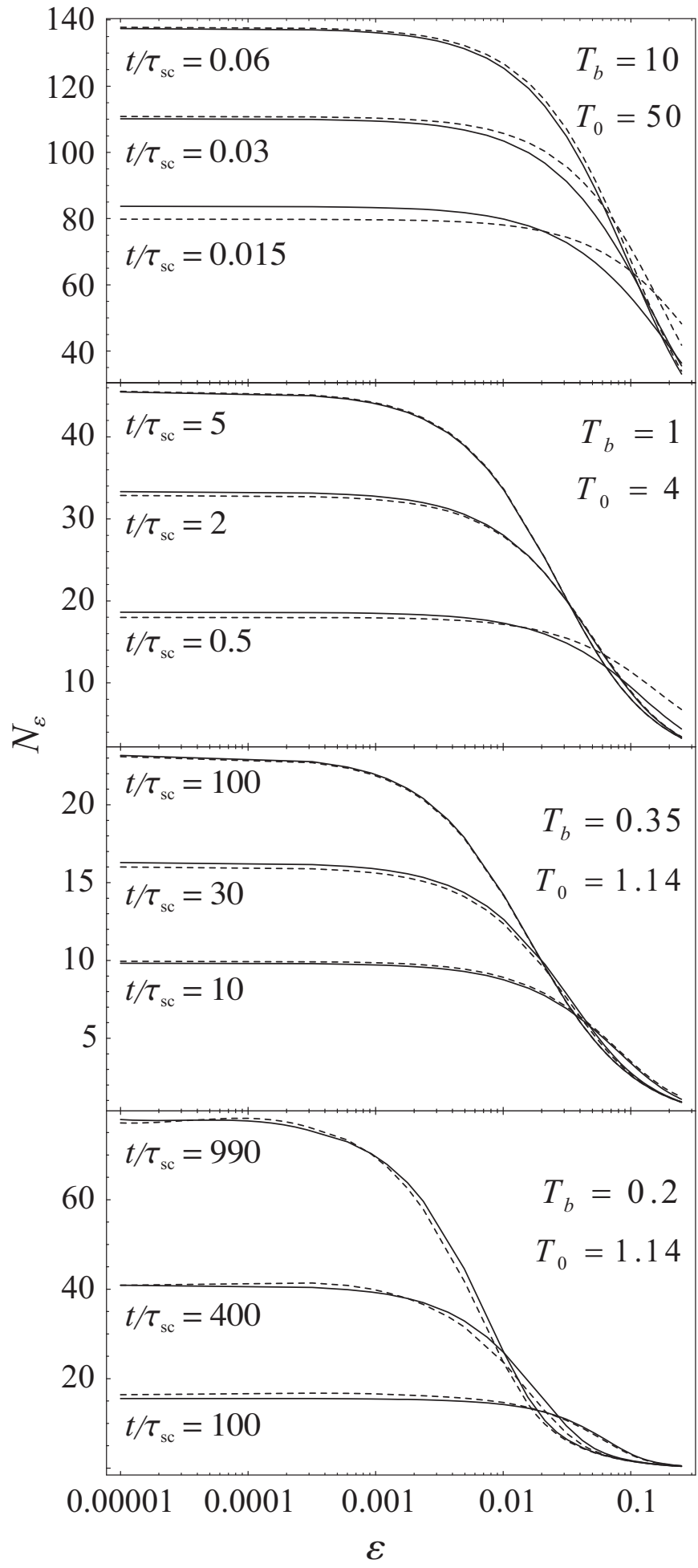

FIG. 6: Evolution of the distribution function $N_{\varepsilon}$ at the adiabatic stage calculated for various sets of the control parameters, $T_{b}$ and $T_{0}$. Solid lines correspond to numerical evaluation of Eq. (2.2); dashed lines are obtained using the generic solution given by Eq. (3.6).
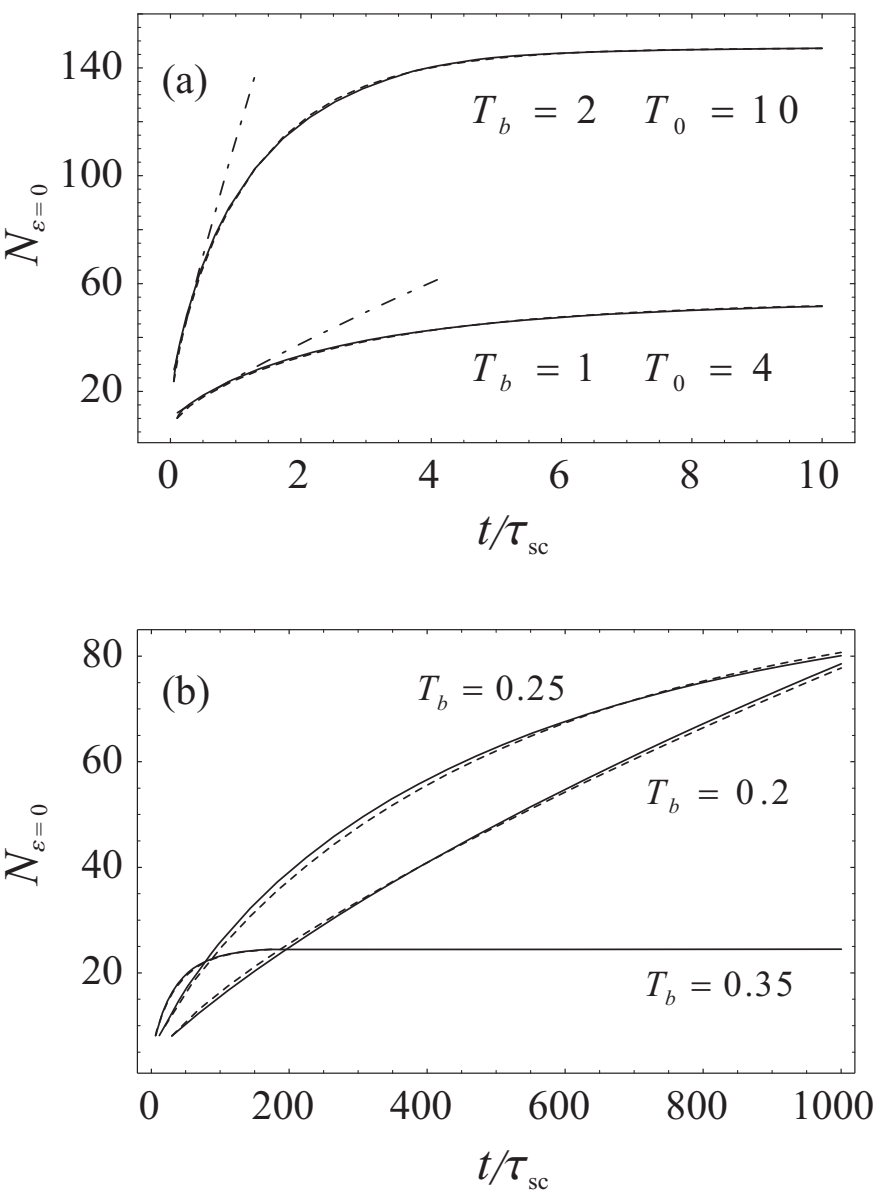

FIG. 7: Population dynamics of the ground state mode $N_{\varepsilon=0}=N_{\varepsilon=0}(t)$ calculated for different bath temperatures at $T_{0}=4,10(\mathrm{a})$, and $T_{0}=1.14$ (b). Similarly to Fig. 6 , the solid and dashed curves are calculated by using Eqs. (2.2) and (3.6), respectively. Dash-dotted lines correspond to time dependences $N_{\varepsilon=0}(t)$ calculated using approximate Eq. (5.1).

so that exciton-exciton interaction eventually becomes the main mechanism of relaxation in GaAs/AlGaAs coupled or ZnSe single QWs.

In contrast, in high-quality GaAs-based microcavities with a relatively large positive detuning $\delta=\hbar\left(\omega_{0}-\omega_{t}\right)$ between the cavity mode $\left(\hbar \omega_{0}\right)$ and QW exciton line $\left(\hbar \omega_{t}\right)$ the LA-phonon-assisted kinetics remains dominant even at relatively high degeneracy temperatures $T_{0} \gg$ $T_{b}>1$. In these systems, by changing the detuning $\delta$ within the band $\delta=50-100 \mathrm{meV}$ one can design an effective in-plane mass $M_{x}$ so that the bottleneck effect in phonon-assisted scattering, due to the low density of states $\propto M_{x}$, is already relaxed, whereas the degeneracy temperature $T_{0} \propto \rho_{2 \mathrm{D}} /\left(E_{0} M_{x}\right)$ is still relatively high. Indeed, in such MCs the lower polariton branch gives rise to the in-plane translational mass much smaller than the mass of optically-undressed QW excitons. In the meantime the excitonic component $\varphi_{X}$ of the microcavity polaritons is already very high, $\varphi_{X} \gtrsim 0.999$, resulting in the long optical decay (in the $z$-direction) lifetimes 


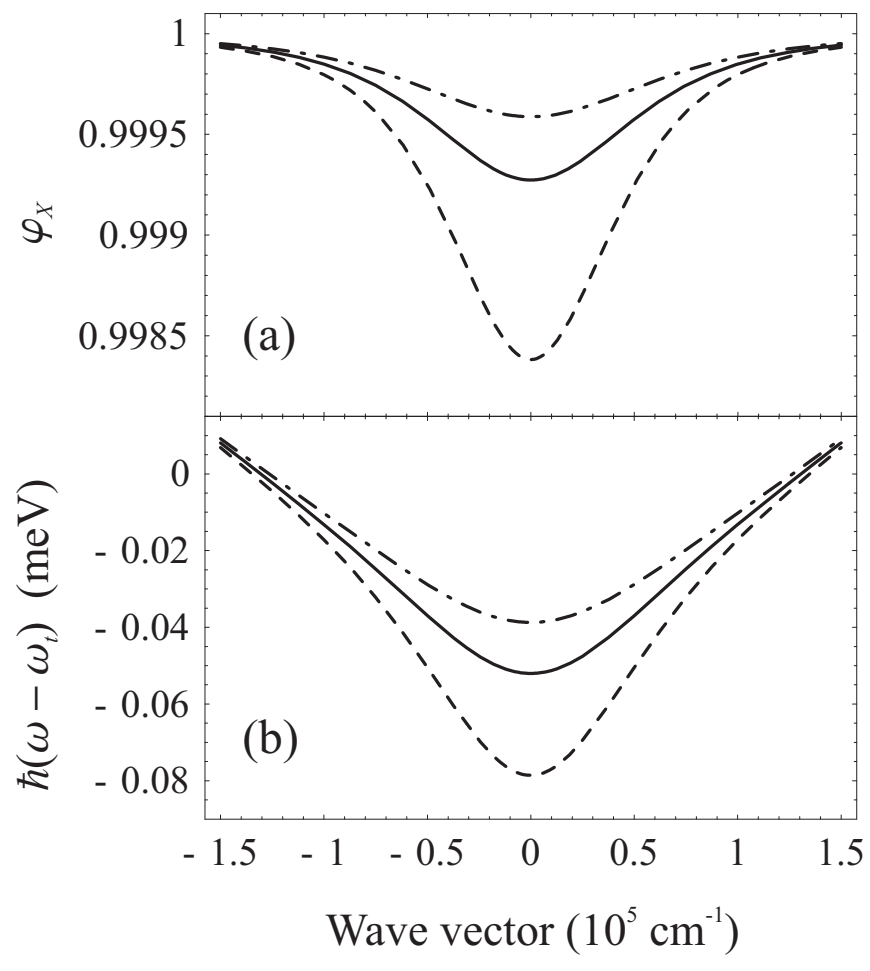

FIG. 8: Possible design of GaAs-based microcavities: (a) excitonic component $\varphi_{X}=\varphi_{X}\left(\mathbf{k}_{\|}\right)$of the MC polariton eigenstate; and (b) the lower branch polariton dispersion $\hbar\left(\omega-\omega_{t}\right)$. Detuning $\delta=50 \mathrm{meV}$ (dashed lines), $75 \mathrm{meV}$ (solid lines), and $100 \mathrm{meV}$ (dash-dotted lines). The energy of ground-state QW excitons is given by $E_{\mathbf{k}_{\|}=0}=\hbar \omega_{t}=1.522 \mathrm{eV}$.

on a few nanosecond time scale. This is illustrated in Fig. 8(a), where the detuning $\delta$ is equal to 50, 75, and 100 meV. The corresponding polariton (exciton) masses are given by $M_{x}=0.023 m_{0}, 0.050 m_{0}$, and $0.081 m_{0}$, respectively. The relevant lower-branch polariton dispersions are plotted in Fig. 8(b). Because the energy $E_{0}$ is only on a $0.01 \mathrm{meV}$ energy scale, the parabolic approximation of the lower-branch dispersion curves is valid for lowtemperature relaxation kinetics. Thus the LA-phononassisted thermalization of the exciton-like MC polaritons can indeed be modelled by the kinetic Eqs. (2.1)-(2.2). Time evolutions of the exciton distribution $N_{\varepsilon}(t)$, which are typical for the proposed design of GaAs-based MCs, are shown in Fig. 6 (see the plots with $T_{b}=1$, and 10). Figure $\mathbb{7}(\mathrm{a})$ illustrates the corresponding population dynamics of the ground-state mode, $N_{\varepsilon=0}=N_{\varepsilon=0}(t)$.

\section{CONCLUSIONS}

In this paper we have studied thermalization kinetics of statistically degenerate QW excitons coupled to thermal bulk acoustic phonons. For concentrations of QW excitons $\rho_{2 \mathrm{D}} \lesssim 10^{9} \mathrm{~cm}^{-2}$ the particle-particle interaction in GaAs or ZnSe QWs can be neglected in comparison with QW exciton - bulk acoustic phonon scattering, and the thermalization kinetics from an initial distribution of QW excitons at $t=0$ occurs through the nonequilibrium states. The following conclusions summarize our results.

(i) For the case of well-developed Bose-Einstein statistics, when $T_{b}<T_{0}$ so that $N_{\varepsilon=0}>1$, the relaxation kinetics of QW excitons coupled to thermal bulk acoustic phonons is given by the following scheme. Within a few characteristic scattering times the correlation of the distribution function $N_{\varepsilon}(t)$ with the initial $N_{\varepsilon}(t=0)$ disappears, and the subsequent thermalization of QW excitons is described in terms of the adiabatic stage of relaxation. The adiabatic stage is characterized by the start time $t_{c}$, which absorbs a gross information about the initial distribution $N_{\varepsilon}(t=0)$, and by the parameter $\lambda_{0}$, which depends only upon the bath and degeneracy temperatures, $T_{b}$ and $T_{0}$. At the beginning of the adiabatic stage, i.e., for the time domain $0 \leq t-t_{c} \lesssim \lambda_{0}^{-1}$, one has $\left|N_{\varepsilon \leq 1 / 4}-N_{\varepsilon \leq 1 / 4}^{0}\right| \simeq N_{\varepsilon \leq 1 / 4}^{0}$, and the thermalization kinetics is strongly nonexponential, with $\delta T \propto 1 / t$ and $N_{\varepsilon=0} \propto(1+\chi t)^{\nu}$. At large times, when the deviation of the system from the final equilibrium state is already small $\left(\left|N_{\varepsilon}(t)-N_{\varepsilon}^{0}\right| / N_{\varepsilon}^{0} \ll 1\right)$, the adiabatic stage of the phonon-assisted thermalization becomes exponential, $\delta T \propto e^{-\lambda_{0} t}$, and can be described within the linearized kinetic equation.

(ii) The linearized LA-phonon-assisted kinetics of QW excitons is formulated in terms of the Fredholm integral Eq. (4.1). The eigenvalues $\{\lambda\}$ of the collision integral are given by the continuous spectrum $\lambda_{0} \leq \lambda<\infty$ and the isolated eigenvalue $\lambda=0$. The marginal eigenvalue $\lambda_{0}=\lambda_{0}\left(T_{b}, T_{0}\right)$ determines the thermalization time at $t \rightarrow \infty$ by $\tau_{\text {th }}=\lambda_{0}^{-1}$. In dependence on the two control parameters, $T_{b}$ and $T_{0} \propto \rho_{2 \mathrm{D}}$, the thermalization time achieves two limits: $\tau_{\text {th }} \ll \tau_{\text {sc }}$ and $\tau_{\text {th }} \gg \tau_{\text {sc }}$. The eigenfunctions $\psi_{\varepsilon}(\lambda)$ of the collision integral (4.1) are smooth integrable functions with three isolated critical points in energy space. The critical points of $\psi_{\varepsilon}(\lambda)$ give rise to a first order pole, a logarithmic singularity, and a continuous step-like jump.

(iii) Because the LA-phonon-assisted kinetics becomes dominant only at small concentrations of QW excitons, $\rho_{2 \mathrm{D}} \lesssim 10^{9} \mathrm{~cm}^{-2}$, nonclassical statistics of quasi-2D excitons in ZnSe or GaAs QWs develops at very low bath temperatures $T_{b}<1 \mathrm{~K}$. The proposed design of GaAsbased microcavities with a relatively large positive detuning between the cavity mode and QW exciton line, $\hbar\left(\omega_{0}-\omega_{t}\right) \geq 50 \mathrm{meV}$, allows us, however, to build up $N_{\varepsilon \simeq 0} \gg 1$ by means of QW exciton - bulk LA phonon scattering in much more favorable conditions, i.e., $T_{b} \gtrsim 1$ $\mathrm{K}$ and $\tau_{\text {th }} \ll \tau_{\text {sc }}$.

\section{Acknowledgments}

We appreciate valuable discussions with L.V. Butov, V. Fal'ko, and M.S. Skolnick. Support of this work by the EPSRC (U.K.) is gratefully acknowledged. 


\section{APPENDIX A: TEMPERATURE LAW}

In Appendix we give the expressions for the parameters and functions used in Eqs. (3.11), (3.15), and (3.16). The parameters $\alpha_{0}$, and $\beta$ arise from those terms in the collision integral which contain $f_{\varepsilon \geq 1 / 4}(t)$ and $f_{\varepsilon \geq 1 / 4}^{2}(t)$, respectively. Collecting such terms together one gets

$$
\begin{aligned}
\alpha_{0}= & \frac{4}{\tau_{\mathrm{sc}} \varepsilon_{c}}\left[\varepsilon_{c} \int_{\varepsilon_{c}-1 / 4}^{\varepsilon_{c}} F_{\mathrm{S}}\left(\varepsilon_{c}, \varepsilon\right)\left(1+n_{\varepsilon}^{\mathrm{ph}}+N_{\varepsilon_{c}-\varepsilon}^{0}\right) \varepsilon d \varepsilon\right. \\
& +\int_{0}^{\varepsilon_{c}-1 / 4} F_{\mathrm{S}}\left(\varepsilon_{c}, \varepsilon\right)\left(1+n_{\varepsilon}^{\mathrm{ph}}+N_{\varepsilon_{c}-\varepsilon}^{0}\right) \varepsilon^{2} d \varepsilon \\
& \left.-\int_{0}^{\infty} F_{\mathrm{AS}}\left(\varepsilon_{c}, \varepsilon\right)\left(n_{\varepsilon}^{\mathrm{ph}}-N_{\varepsilon_{c}+\varepsilon}^{0}\right) \varepsilon^{2} d \varepsilon\right], \quad(\mathrm{A} 1)
\end{aligned}
$$

and

$$
\begin{aligned}
\beta= & \frac{4}{\tau_{\mathrm{sc}} T_{b}}\left[\int_{0}^{\varepsilon_{c}-1 / 4} F_{\mathrm{S}}\left(\varepsilon_{c}, \varepsilon\right)\left(N_{\varepsilon_{c}-\varepsilon}^{0}\right)^{\prime}\left(\varepsilon_{c}-\varepsilon\right) \varepsilon d \varepsilon\right. \\
& \left.-\int_{0}^{\infty} F_{\mathrm{AS}}\left(\varepsilon_{c}, \varepsilon\right)\left(N_{\varepsilon_{c}+\varepsilon}^{0}\right)^{\prime}\left(\varepsilon_{c}+\varepsilon\right) \varepsilon d \varepsilon\right] .
\end{aligned}
$$

The function $\alpha_{1}(t)$ stems from the terms proportional to $f_{\varepsilon \geq 1 / 4}(t) f_{\varepsilon<1 / 4}(t)$ and is given by

$$
\alpha_{1}(t)=\frac{4 T_{b}}{\tau_{\mathrm{sc}}} \int_{0}^{1 / 4} F_{\mathrm{S}}\left(\varepsilon_{c}, \varepsilon_{c}-\varepsilon\right)\left(N_{\varepsilon}^{0}\right)^{\prime} f_{\varepsilon}(t)\left(\varepsilon_{c}-\varepsilon\right) d \varepsilon .
$$

The parameter $\tilde{\alpha}$ from Eq. (3.13) is obtained by collecting all the terms $\propto \delta T(t)$ :

$$
\begin{aligned}
\tilde{\alpha}= & -\int_{\varepsilon_{c}-1 / 4}^{\varepsilon_{c}} F_{\mathrm{S}}\left(\varepsilon_{c}, \varepsilon\right)\left(1+n_{\varepsilon}^{\mathrm{ph}}+N_{\varepsilon_{c}-\varepsilon}^{0}\right) \frac{\eta\left(\varepsilon_{c}-\varepsilon\right)}{\xi_{0}\left(\varepsilon_{c}-\varepsilon\right)} \varepsilon d \varepsilon \\
& -\left(\alpha_{0} \tau_{\mathrm{sc}} \varepsilon_{c}\right) /\left(4 T_{b}^{2}\right) .
\end{aligned}
$$

When deriving Eq. (3.16) from Eq. (3.15) we first put $\varepsilon=0$ everywhere in the integrand (3.15) except the exponent. By changing the integration variable $\varepsilon$ to $\xi=\xi_{0}(\varepsilon)$ and putting $\varepsilon=0$ we derive the asymptotic Eq. (3.16), valid in the limit $t \rightarrow \infty$. In this equation $\tilde{\gamma}_{0}$ is a timeindependent pre-factor given by

$$
\tilde{\gamma}_{0}=F_{\mathrm{S}}\left(\varepsilon_{c}, \varepsilon_{c}\right)\left(1+n_{\varepsilon_{c}}^{\mathrm{ph}}+N_{\varepsilon=0}^{0}\right) \frac{\varepsilon_{c} f_{\varepsilon=0}\left(t_{0}\right)}{\xi_{0}^{\prime}(0)} .
$$

* e-mail: Alexander.Soroko@astro.cf.ac.uk

1 For a review of the experimental works on relaxation and photoluminescence kinetics of QW excitons see, e.g., J. Shah, Ultrafast Spectroscopy of Semiconductors and Semiconductor Nano-structures, Springer Series in Solid-State Sciences, Vol. 115 (Springer, Berlin, 1996).

2 T. Takagahara, Phys. Rev. B 31, 6552 (1985).

3 A. Vinattieri, J. Shah, T.C. Damen, D.S. Kim, L.N. Pfeiffer, M.Z. Maialle, and L.J. Sham, Phys. Rev. B 50, 10868 (1994).

4 P.E. Selbmann, M. Gulia, F. Rossi, E. Molinari, and P. Lugli, Phys. Rev. B 54, 4660 (1996).

5 M. Gurioli, P. Borri, M. Colocci, M. Gulia, F. Rossi, E. Molinari, P.E. Selbmann, and P. Lugli, Phys. Rev. B 58, R13403 (1998).

6 M. Gulia, F. Rossi, E. Molinari, P.E. Selbmann, and P. Lugli, Phys. Rev. B 55, R16049 (1997).

7 I.K. Oh, J. Singh, A. Thilagam, and A.S. Vengurlekar, Phys. Rev. B 62, 2045 (2000).

8 A.L. Ivanov, P.B. Littlewood, and H. Haug, Phys. Rev. B 59, 5032 (1999).

9 T.C. Damen, J. Shah, D.Y. Oberli, D.S. Chemla, J.E. Cunningham, and J.M. Kuo, Phys. Rev. B 42, 7434 (1990).

10 R. Eccleston, R. Strobel, W.W. Rühle, J. Kuhl, B.F. Feuerbacher, and K. Ploog, Phys. Rev. B 44, 1395 (1991); R. Eccleston, B.F. Feuerbacher, J. Kuhl, W.W. Rühle, and
K. Ploog, Phys. Rev. B 45, 11403 (1992).

11 T. Fukuzawa, E.E. Mendez, and J.M. Hong, Phys. Rev. Lett. 25, 3066 (1990). However, the experimental results of this work were later reinterpreted.

12 L.V. Butov, A. Zrenner, G. Abstreiter, G. Böhm, and G. Weimann, Phys. Rev. Lett. 73, 304 (1994).

13 L.V. Butov and A.I. Filin, Phys. Rev. B 58, 1980 (1998).

14 L.V. Butov, A. Imamoglu, A.V. Mintsev, K.L. Campman, and A.C. Gossard, Phys. Rev. B 59, 1625 (1999).

15 L.V. Butov, A.L. Ivanov, A. Imamoglu, P.B. Littlewood, A.A. Shashkin, V.T. Dolgopolov, K.L. Campman, and A.C. Gossard, Phys. Rev. Lett. 86, 5608 (2001).

16 A.V. Larionov and V.B. Timofeev, Pis'ma Zh. Eksp. Teor. Fiz. 73, 342 (2001) [JETP Lett. 73, 301 (2001)].

17 V.V. Krivolapchuk, E.S. Moskalenko, and A.L. Zhmodikov, Phys. Rev. B 64, 045313 (2001).

18 S. Charbonneau, M. L. W. Thewalt, E.S. Koteles, and B. Elman, Phys. Rev. B 38, 6287 (1988).

19 A. Alexandrou, J.A. Kash, E.E. Mendez, M. Zachau, and J.M. Hong, Phys. Rev. B 42, 9225 (1990).

20 J.E. Golub, K. Kash, J.P. Harbison, and L.T. Florez, Phys. Rev. B 41, 8564 (1990).

21 J.E. Golub, K. Kash, J.P. Harbison, and L.T. Florez, Phys. Rev. B 45, 9477 (1992).

${ }^{22}$ F. Clérot, B. Deveaud, A. Chromette, A. Regreny, and B. Sermage, Phys. Rev. B 41, 5756 (1990). 
23 A. Parlangeli, P.C.M. Christianen, J.C. Maan, I.V. Tokatly, C.B. Soerensen, and P.E. Lindelof, Phys. Rev. B 62, 15323 (2000).

24 M. Umlauff, J. Hoffmann, H. Kalt, W. Langbein, J.M. Hvam, M. Scholl, J. Söllner, M. Heuken, B. Jobst, and D. Hommel, Phys. Rev. B 57, 1390 (1998); H. Kalt, J. Hoffmann, M. Umlauff, W. Langbein, and J.M. Hvam, Phys. Status Solidi B 206, 103 (1998).

25 G. Bacher, R. Spiegel, T. Kümmell, R. Weigand, A. Forchel, B. Jobst, D. Hommel, and G. Landwehr, J. Cryst. Growth 184/185, 330 (1998).

26 E.A. Uehling and G.E. Uhlenbeck, Phys. Rev. 108, 1175 (1932).

27 U. Bockelmann, Phys. Rev. B 50, 17271 (1994).

28 Yu.E. Lozovik and V.I. Yudson, Pis'ma Zh. Eksp. Teor. Fiz. 22, 556 (1975) [JETP Lett. 22, 274 (1975)].

29 X. Zhu, P.B. Littlewood, M. Hybertsen, and T. Rice, Phys. Rev. Lett. 74, 1633 (1995).

30 P.B. Littlewood and X. Zhu, Physica Scripta 68, 56 (1996).

31 Y. Naveh and B. Laikhtman, Phys. Rev. Lett. 77, 900 (1996).

32 P.G. Savvidis, J.J. Baumberg, R.M. Stevenson, M.S. Skolnick, D.M. Whittaker, and J.S. Roberts, Phys. Rev. Lett. 84, 1547 (2000).

33 A.I. Tartakovskii, M. Emam-Ismail, R.M. Stevenson, M.S. Skolnick, V.N. Astratov, D.M. Whittaker, J.J. Baumberg, and J.S. Roberts, Phys. Rev. B 62, R2283 (2000)

${ }^{34}$ F. Boeuf, R. André, R. Romestain, Le Si Dang, E. Péronne, J.F. Lampin, D. Hulin, and A. Alexandrou, Phys. Rev. B 62, R2279 (2000).

35 R.M. Stevenson, V.N. Astratov, M.S. Skolnick, D.M. Whittaker, M. Emam-Ismail, A.I. Tartakovskii, P.G. Savvidis, J.J. Baumberg, and J.S. Roberts, Phys. Rev. Lett. 85, 3680 (2000).

36 J.J. Baumberg, P.G. Savvidis, R.M. Stevenson, A.I. Tartakovskii, M.S. Skolnick, D.M. Whittaker, and J.S. Roberts, Phys. Rev. B 62, R16247 (2000).

37 P.G. Savvidis, C. Ciuti, J.J. Baumberg, D.M. Whittaker, M.S. Skolnick, and J.S. Roberts, Phys. Rev. B 64, 075311 (2001).

38 S.D. Baranovskii, R. Eichmann, and P. Thomas, Phys. Rev. B 58, 13081 (1998).

39 C. Bradford, C. B. O’Donnell, B. Urbaszek, C. Morhain, A. Balocchi, K. A. Prior, and B. C. Cavenett, Phys. Rev. B 64, 195309 (2001).

40 A.L. Ivanov, C. Ell, and H. Haug, Phys. Rev. E 55, 6363 (1997); Phys. Status Solidi B 206, 235 (1998).

41 C. Cercignani, The Boltzmann Equation and Its Applications, Springer Series in Applied Mathematical Sciences Vol. 67 (Springer, New York, 1988), Sect. IV-6, pp. 180189. 